\title{
Review Article \\ Sclerostin Antibody Therapy for the Treatment of Osteoporosis: Clinical Prospects and Challenges
}

\author{
Claire MacNabb, D. Patton, and J. S. Hayes \\ Regenerative Medicine Institute, NUI Galway, Biosciences Research Building, Corrib Village, Dangan, Galway, Ireland \\ Correspondence should be addressed to J. S. Hayes; jessica.hayes@nuigalway.ie
}

Received 23 February 2016; Accepted 21 April 2016

Academic Editor: Merry Jo Oursler

Copyright ( 2016 Claire MacNabb et al. This is an open access article distributed under the Creative Commons Attribution License, which permits unrestricted use, distribution, and reproduction in any medium, provided the original work is properly cited.

\begin{abstract}
It is estimated that over 200 million adults worldwide have osteoporosis, a disease that has increasing socioeconomic impact reflected by unsustainable costs associated with disability, fracture management, hospital stays, and treatment. Existing therapeutic treatments for osteoporosis are associated with a variety of issues relating to use, clinical predictability, and health risks. Consequently, additional novel therapeutic targets are increasingly sought. A promising therapeutic candidate is sclerostin, a Wnt pathway antagonist and, as such, a negative regulator of bone formation. Sclerostin antibody treatment has demonstrated efficacy and superiority compared to other anabolic treatments for increasing bone formation in both preclinical and clinical settings. Accordingly, it has been suggested that sclerostin antibody treatment is set to achieve market approval by 2017 and aggressively compete as the gold standard for osteoporotic treatment by 2021. In anticipation of phase III trial results which may potentially signify a significant step in achieving market approval here, we review the preclinical and clinical emergence of sclerostin antibody therapies for both osteoporosis and alternative applications. Potential clinical challenges are also explored as well as ongoing developments that may impact on the eventual clinical application of sclerostin antibodies as an effective treatment of osteoporosis.
\end{abstract}

\section{Introduction}

The identification of sclerostin as a therapeutic target and the optimisation of anti-sclerostin antibodies (Scl-mAb) have led to a vast array of preclinical studies documenting its ability to enhance bone formation, strength, and density $[1,2]$. Developments have even attracted the attention of NASA with treatment potentially capable of reversing bone density deterioration experienced by astronauts during prolonged space flight [3]. The first human clinical trial continued the success story with a single injection of Scl-mAb surpassing gains in bone mineral density beyond levels expected after six months of daily teriparatide injections [4]. Amgen (romosozumab), Eli Lilly (blosozumab), and Novartis (BPS804) represent the main industrial backers of Scl-mAb therapy. In light of recent clinical trial developments, industry commentators fully expect market approval for a humanized anti-sclerostin antibody by 2017 [5]. There is little doubt that there is a serious need for effective treatment. The World Health Organization considers osteoporosis second only to cardiovascular disease as a threat to global health with over 200 million sufferers globally facing an increased risk of fracture and related complications [6]. Current approaches encompass largely anti-resorptive treatments such as bisphosphonates, selective oestrogen receptor modulators, oestrogen, and denosumab antibody therapy. Teriparatide available as either full-length (recombinant human PTH 1-84) or the active fragment (PTH 1-34) is currently the only clinically available anabolic treatment for osteoporosis. However, only PTH 1-34 is licensed for use in the USA. Moreover, there are considerable disadvantages with the use of teriparatide. For instance, the use of teriparatide and recombinant human PTH has been limited to 18-24 months in the USA and EU, respectively. Also, while initially associated with increases in bone formation eventually their use leads to a rise in markers of bone resorption. Consequently, while many existing therapies are available, new treatment options are continuously sought. In this review, we provide a perspective of the field of Scl-mAb therapy. Specifically, we examine the development of Scl$\mathrm{mAb}$ through preclinical and clinical studies while assessing 
the strengths and potential short-comings of treatments. A commentary on areas for further research and novel future applications is also explored.

\section{Osteoporosis and the Need for Intervention}

Osteoporosis is a metabolic bone disease characterised by a significant decrease in bone mineral density (BMD) and structural changes to the bone that greatly increase the risk of fracture [7]. The condition is age-related and affects both sexes. However, it is particularly prevalent in postmenopausal women with one in three women aged over 50 likely to experience an osteoporotic fracture compared to one in five men. The increased prevalence after menopause is due to decreased oestrogen levels which accelerate bone loss. Hip fractures, in particular, have a serious impact on patient welfare. These result in reduced mobility, chronic pain, and a greatly increased level of dependence, with $10-20 \%$ of previously independent sufferers being admitted to nursing homes [8]. The social and economic cost of osteoporosis is considerable. It is estimated that 1.6 million osteoporotic hip fractures occur annually and are set to increase to 6.3 million globally by 2050 [9]. Within the EU in 2010, approximately 5.5 million men and 22 million women were estimated to have osteoporosis. Moreover, the cost of treatment for osteoporotic fractures and pharmaceutical intervention of the disease are projected at $€ 37$ billion per annum [8]. Osteoporotic fractures are further complicated by the inherent difficulties in ensuring adequate fixation of pins and screws due to diminished quality of the surrounding bone $[10,11]$. The occurrence of osteoporosis is set to increase drastically with the number of sufferers in the EU alone forecasted to rise by $23 \%$ to 33.9 million by 2025 [12]. This will create a serious medical and economic challenge and highlights the need for effective strategies to deal with the issue on a global scale [13]. In order to be successful, the development of these international strategies must be built on a solid foundation of safe and effective therapies that can be applied at an individual level.

\section{Sclerostin, Its Mode of Action and Potential as a Therapeutic Target}

3.1. Sclerostin. The role of sclerostin in the modulation of the Wnt $\beta$-catenin dependent pathway came to light specifically through the study of sclerosteosis and van Buchem disease, two rare genetic disorders associated with high levels of BMD and an associated low risk of fracture [14]. Both diseases were traced to mutations impacting a single gene, SOST, which is mainly expressed from osteocytes. Nevertheless, its expression in chondrocytes, osteoblasts, the bone marrow, heart, pancreas, liver, and some foetal tissue has been reported [1517]. The glycoprotein sclerostin is a product of the SOST gene. The amino acid sequence of sclerostin distantly resembles that of the Cerebus/DAN (differential screening-selected gene aberrative in neuroblastoma) family of glycoproteins, defined by 190 residues with a cysteine knot-like domain. However, while DAN proteins act as classical antagonists of BMP signalling, sclerostin-BMP interactions are weak and appear to be specific to BMP-7 in osteocytes alone [18, 19]. Others suggest that sclerostin exhibits a catabolic effect on bone by increasing osteoclast production via increased osteocytic expression of receptor activator of nuclear factor kappa B (RANKL). The decline in RANKL signalling may at least in part explain the sustained decreases in bone resorption markers observed with antisclerostin therapy [20, 21].

Several sources of evidence implicate a prominent role for sclerostin as an antagonist of the Wnt signalling pathway $[22,23]$. The Wnt gene family are largely characterised by their highly conserved glycosylated secreted proteins and can be broadly defined by canonical and noncanonical mediators. The latter typically involve cGMP-related signalling, Junkinase activation, and/or activation of protein kinase A. Primarily the noncanonical pathway is involved in processes such as tissue formation during development, maintenance of adult stem cells, and tumour repression [24]. Canonical Wnts are defined by their ability to stabilise $\beta$-catenin via Wnt ligand binding to the Frizzled (FZD) receptor and low-density lipoprotein receptor-related proteins 5 and 6 (LRP5/6). This results in the phosphorylation of LRP5/6 thus permitting Axin to bind to the receptor complex. This results in the inhibition of glycogen synthase kinase- $3 \beta$ 's (GSK-3 $\beta$ ) activity, which ordinarily targets $\beta$-catenin for degradation. However, the creation of this complex stabilises cytosolic $\beta$-catenin resulting in its nuclear translocation. Within the nucleus, it induces downstream transcription of bone-related genes such as Runx2 and osteocalcin via T-cell factor/lymphoid enhancer binding factor (TEF/LEF) cofactors [25-27] (Figure 1(a)). Activation of the Wnt/ $\beta$ catenin dependent pathway also modulates osteogenic and chondrogenic differentiation of mesenchymal stem cells as well as regulating bone mass by increasing osteoprotegerin expression thereby reducing osteoclastogenesis [28, 29]. It has been shown that sclerostin exerts its negative regulation of bone formation by binding via its central core to the extracellular domains LPR5/6 at the first $\beta$-propeller via an NXI motif. This prevents Wnt and FZD ligand binding $[30,31]$. Fundamentally, the interference of sclerostin in the Wnt/LRP/FZD complex results in uninhibited GSK-3 $\beta$ activity and the phosphorylation of $\beta$-catenin leading to its subsequent degradation. Consequently, translocation into the nucleus is not actively facilitated which renders Wnt pathway gene promoters inactivated. This effectively inhibits the anabolic function of Wnt signalling for bone and as a consequence decreases bone formation [32] (Figure 1(b)).

3.2. Sclerostin as a Therapeutic Target. Mutations in the LRP receptors initially helped establish a link between Wnt signalling and disease and their role as positive regulators of bone formation. The LRP5 gene was initially identified as a determinant of bone mass in the 1990s. Specifically, linkage analyses studies demonstrated that the human chromosome $11 q 13$ was associated with two extremes of low and high bone mass [33, 34]. Osteoporosis pseudoglioma syndrome, an autosomal recessive hereditary disorder (characterised by low bone mass and abnormal eye vasculature), was traced to an inactivation mutation of LRP5 [33]. In contrast, gainof-function LRP5 mutations are linked to the autosomal 


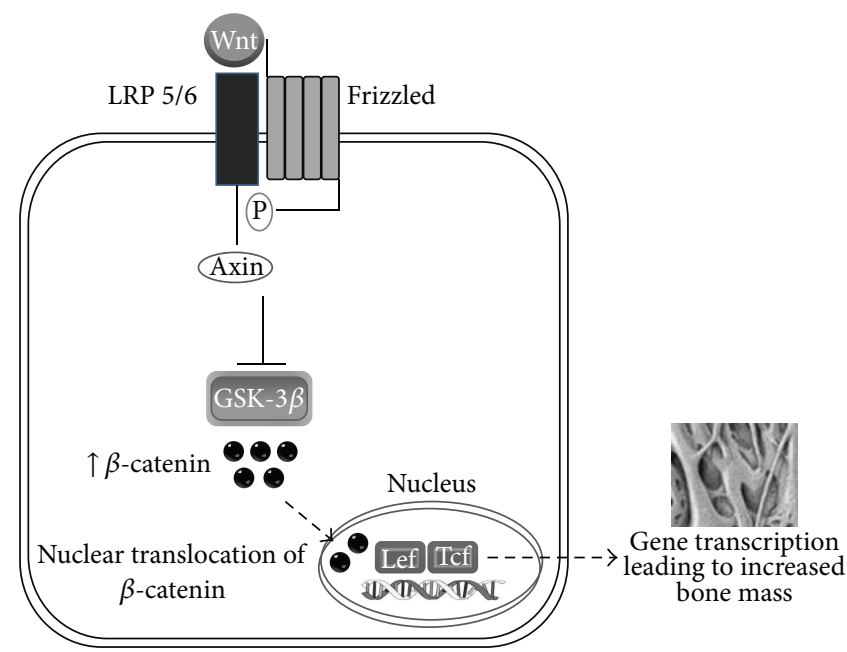

(a)

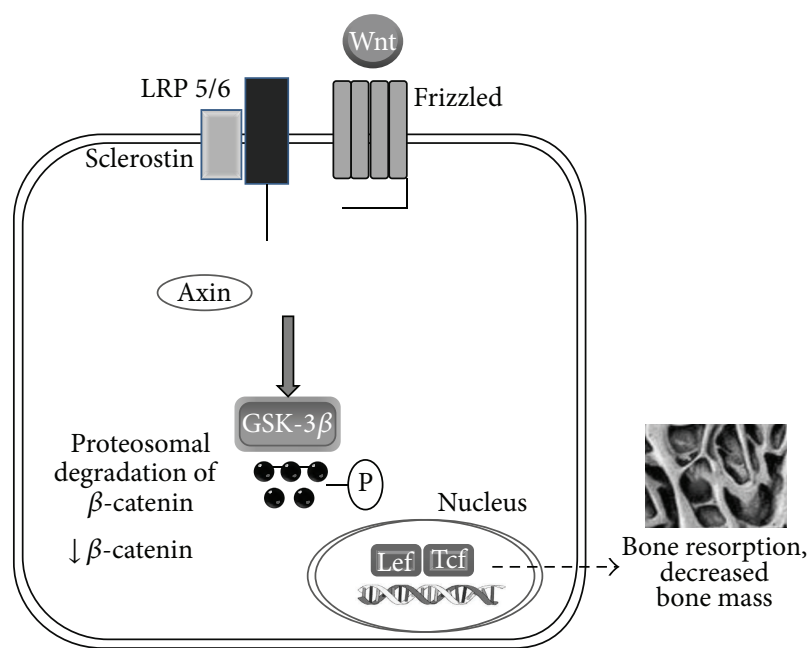

(b)

Figure 1: (a) Canonical Wnt signalling involves binding of Wnt to LRP5/6 and its coreceptor Frizzled resulting in the phosphorylation of LRP5/6 thus permitting Axin to bind to the receptor complex. Formation of this complex leads to inhibition of GSK3 $\beta$ which prevents degradation of $\beta$-catenin. Accumulation of cytosolic $\beta$-catenin leads to nuclear translocation where it activates target gene promoters which result in increased bone mass. (b) Sclerostin inhibition of the Wnt-canonical pathway in osteogenesis. Sclerostin binding to LRP receptor 5/6 prevents Wnt binding and formation of the Frizzled-LRP complex and thus Axin remains unphosphorylated. Downstream effects include activation of GSK $3 \beta$ resulting in phosphorylation of cytosolic $\beta$-catenin, thus targeting it for degradation. In the absence of $\beta$-catenin accumulation and subsequent nuclear translocation, osteogenesis is prevented.

dominant high bone mass (HBM) which are defined by excessive bone formation, bone thickening, and reduced risk of fracture $[34,35]$. These findings were confirmed in mouse models of defective Wnt signalling. Briefly, LRP5-deficient mice manifested an osteoporotic phenotype arising from the impaired bone formation while bone resorption remained unaffected. In contrast, mice carrying an HBM mutation within the LRP5 gene display osteosclerosis [36, 37]. Several studies using Cre-loxP-technology focused on the inactivation of $\beta$-catenin specifically in osteoblasts and osteoclasts in an effort to understand the molecular signature of the skeletal phenotype displayed by these diseases. Essentially, these and other similar studies demonstrated that $\beta$-catenin deletion in cells within the osteoclast lineage resulted in increased osteoclastogenesis. In contrast, $\beta$-catenin deletion in mesenchymal osteoprogenitor cells resulted in the prevention of osteoblast differentiation with an associated preference for chondrogenesis $[38,39]$. Importantly, $\beta$-catenin inactivation in fully differentiated osteoblasts and osteocytes resulted in increased osteoclastogenesis arising from the decreased osteoprotegerin while bone formation remained unaffected $[29,40,41]$.

More recently it has been demonstrated that Frizzled-8 and $\beta$-catenin negatively regulate osteoclast differentiation independent of osteoblasts and that canonical Wnt signalling controls bone resorption by two different mechanisms [42]. The authors report that mice deficient in Frizzled- 8 manifest osteopenia associated with the unhindered bone formation and increased osteoclastogenesis. Nevertheless, this phenotype was not associated with impaired osteoprotegerin production or Wnt signalling by osteoblasts. Further, $\beta$ catenin deletion in the osteoclast lineage confirmed the negative influence of canonical Wnt signalling on osteoclastogenesis. Here increased bone resorption was evident despite the apparently normal production of osteoprotegerin by osteoblasts being observed [42]. The reader is directed to a recent review for a more comprehensive insight into the molecular associations of LRP5 and bone formation [43]. The potential of sclerostin as a therapeutic target was soon recognised and gained momentum when animal studies with SOST knock-out mice were also found to have increased BMD over wild-type counterparts, whereas transgenic mice overexpressing SOST displayed an osteoporotic bone phenotype $[18,44]$. Given the similarities to the changes observed in LRP5 mutations, the notion sclerostin functioned as an LRP5 antagonist was pursued and later confirmed. Nonetheless, it is now recognised that other interactions are also involved in the antiosteoanabolic role of sclerostin $[22,23]$. More recently, Yorgan and colleagues attempted to clarify if sclerostin truly acts as a Wnt signalling antagonist by interacting with LRP5. The authors generated Collal-SOST mice with transgenic overexpression of sclerostin under the control of a 2.3-kb Collal promoter, resulting in a low bone mass phenotype. The two mouse lines used carried different high bone mass mutations of LRP5 (LRP5 (A170V) and LRP5 $(\mathrm{G} 213 \mathrm{~V}))$. Subsequently, the authors found that the inhibitory function of sclerostin overexpression on bone formation was not observed in LRP5 (G213V/G213V) mice and was strongly reduced in LRP5 (A170V/A170V) mice. The authors then adopted a similar approach whereby the transmembrane Wnt signalling antagonist Krm2 was overexpressed in mice. Interestingly, the antiosteoanabolic influence of the CollalKrm2 transgene was not affected by either LRP5 mutation investigated [43]. 
Due the heterogeneous cellular make-up of bone, gaining detailed insight into the transcriptional response to Scl-mAb and the associated mechanistic nature of its modulation of Wnt signalling pathway has proved challenging. In the context of the mesenchymal stem cell osteogenic differentiation pathway, sclerostin activity has the most effect on the late osteoblast cells, inhibiting the terminal differentiation of osteoblasts and the associated mineral deposition. It has been postulated that sclerostin may function in the upregulation of small integrin-binding ligand N-glycoprotein (SIBLING) family formation. SIBLING proteins bind to newly mineralised surfaces preventing further mineralisation through acidic serine aspartate-rich motifs (ASARM peptides). It also appears that the formation of PHEX, a metalloprotease capable of inactivating ASARM peptides disruption of mineralisation, is inhibited by sclerostin too [45]. More recently, Nioi and colleagues used laser capture microdissection to assess changes in mRNA expression of specific canonical Wnt-related genes in osteoblasts, osteocytes, and bone lining cells in ovx-rat vertebrae that had undergone a single administration $(100 \mathrm{mg} / \mathrm{kg})$ of $1 \mathrm{Scl}-\mathrm{mAb}$. Samples were obtained 6, 24, 72, and 168 hours after Scl-mAb administration [46]. Microarray analyses revealed that five canonical Wnt-related genes in particular, namely, Gjal, Bglap, Twistl, Mmp2, and Wisp1, were markedly upregulated subsequent to $\mathrm{Scl}-\mathrm{mAb}$ exposure, suggesting a targeted activation of canonical Wnt signalling associated genes. Notably, there was a significant upregulation of extracellular matrix-associated genes in osteoblasts, osteocytes, and bone lining cells in response to $\mathrm{Scl}-\mathrm{mAb}$ treatment. This would suggest that bone anabolism is facilitated via the activation of matrixproducing osteoblasts and transition of bone lining cells to active matrix-producing osteoblasts. Interestingly, while Scl-mAb treatment has been observed in several animal and human studies to enhance bone formation and reduce bone resorption, here gene modulation of osteoclastogenesis remained unaffected. Increased osteoprotegerin is a likely candidate modulator given it is recognised as a canonical Wnt target. However, the authors did not observe any changes in expression [46]. The complexity of the mechanisms by which Scl-mAb exert their effect is an ongoing area of research and vital to a full understanding of its potential as a therapeutic target. Meanwhile, the neutralisation of sclerostin using monoclonal antibodies has been subject to numerous preclinical studies and a range of clinical trials which are addressed below.

\section{Current Clinical Trial Data Relating to Anti-Sclerostin Monoclonal Antibody Therapy}

The literature on sclerostin and its inhibition with monoclonal antibodies has been well reviewed. These reviews include its discovery and development as a therapeutic target, its mode of action, and its suitability as a biochemical marker, along with more general overviews [2, 47-49]. The initial development and screening of sclerostin neutralising antibodies involved a murine IgG1 produced in a hybridoma cell line which was validated in vitro [50]. Due to the subsequent commercial development of the antibody, information relating to its characteristics has been limited. Nevertheless, the Eli Lilly anti-sclerostin antibody blosozumab is described as an IgG4 antibody [21] while the Novartis humanized Scl-mAb is described as an IgG2 antibody. The first in vivo study to test anti-sclerostin antibodies for increasing bone mass involved a rodent model of postmenopausal osteoporosis (Figure 2). Ovariectomised (ovx) rats were left untreated for one year in order to develop significant levels of bone loss. Treatment of 19 -month-old ovx-rats with Scl-mAb (25 mg/kg, biweekly for 5 weeks) appeared to completely reverse the bone loss exhibited over the previous year-long period. In fact, bone strength and mass were both reportedly higher in Scl-mAb treated ovx-rats compared to non-ovx control animals [50]. More recently, it has been determined that the increases in bone formation observed due to Scl-mAb treatment in rats and primates do not negatively influence bone matrix quality even where increases in bone volume as high as $54 \%$ are noted [51]. The transition from preclinical models to human clinical trials was facilitated by a major primate study that used dosage levels and a delivery schedule intended to replicate those of early-stage human trials. This study confirmed a strong correlation between serum antibody levels and serum levels of the bone formation markers osteocalcin and P1NP and was considered to provide strong evidence that the effect of treatment was robust and reproducible [52]. A complete chronological summary of all clinical trials investigating Scl$\mathrm{mAb}$ is presented in Table 1 .

4.1. Romosozumab. Data from two phase I trials of the humanized Scl-mAb romosozumab (Amgen, formally known as AMG785) have been published. The initial first-in-human single-dose randomized study included healthy men and postmenopausal women (aged 45-59; NCT01059435). This was followed by a multidose study (NCT01825785) [4, 53]. Padhi et al. describe a randomized, double-blind, placebocontrolled, ascending-single-dose study in healthy men and postmenopausal women which received romosozumab or placebo $(3: 1)$ subcutaneously $(0.1,0.3,1,3,5$, or $10 \mathrm{mg} / \mathrm{kg})$ or intravenously ( 1 or $10 \mathrm{mg} / \mathrm{kg}$ ). The primary objective of the study was to establish safety and tolerability of romosozumab. Secondary objectives included the evaluation of pharmacokinetics, bone turnover markers, and bone mineral density. A total of 72 subjects were enrolled with 56 (14 placeboes, 42 romosozumab) receiving investigational product administered subcutaneously while 16 subjects (4 placeboes, 12 romosozumab) received the investigational product intravenously.

Both subcutaneous and intravenous dosing resulted in a greater-than-dose-proportional increase in romosozumab serum concentrations, with apparent clearance decreasing as dose increased. Increases in P1NP, bone-specific alkaline phosphatase (BAP) and osteocalcin (markers of bone formation) were dose-related with maximum increases from baseline of $184 \%, 126 \%$, and $176 \%$, respectively. The serum concentration of C-terminal telopeptide of type 1 collagen (CTx; bone resorption marker) concomitantly decreased in an approximate dose-dependent manner with a maximum decrease of $54 \%$ below baseline reported. As expected, BMD 


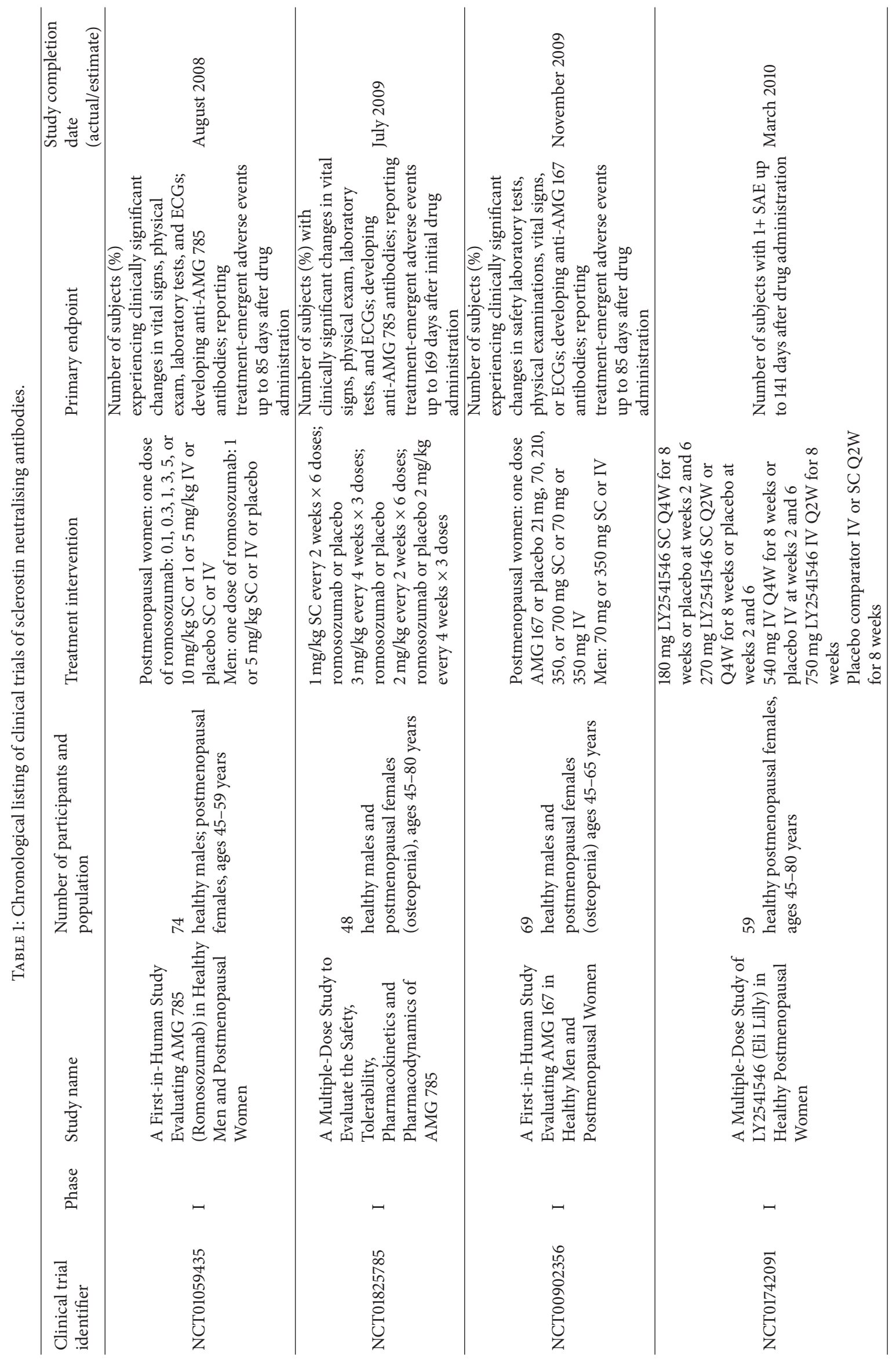




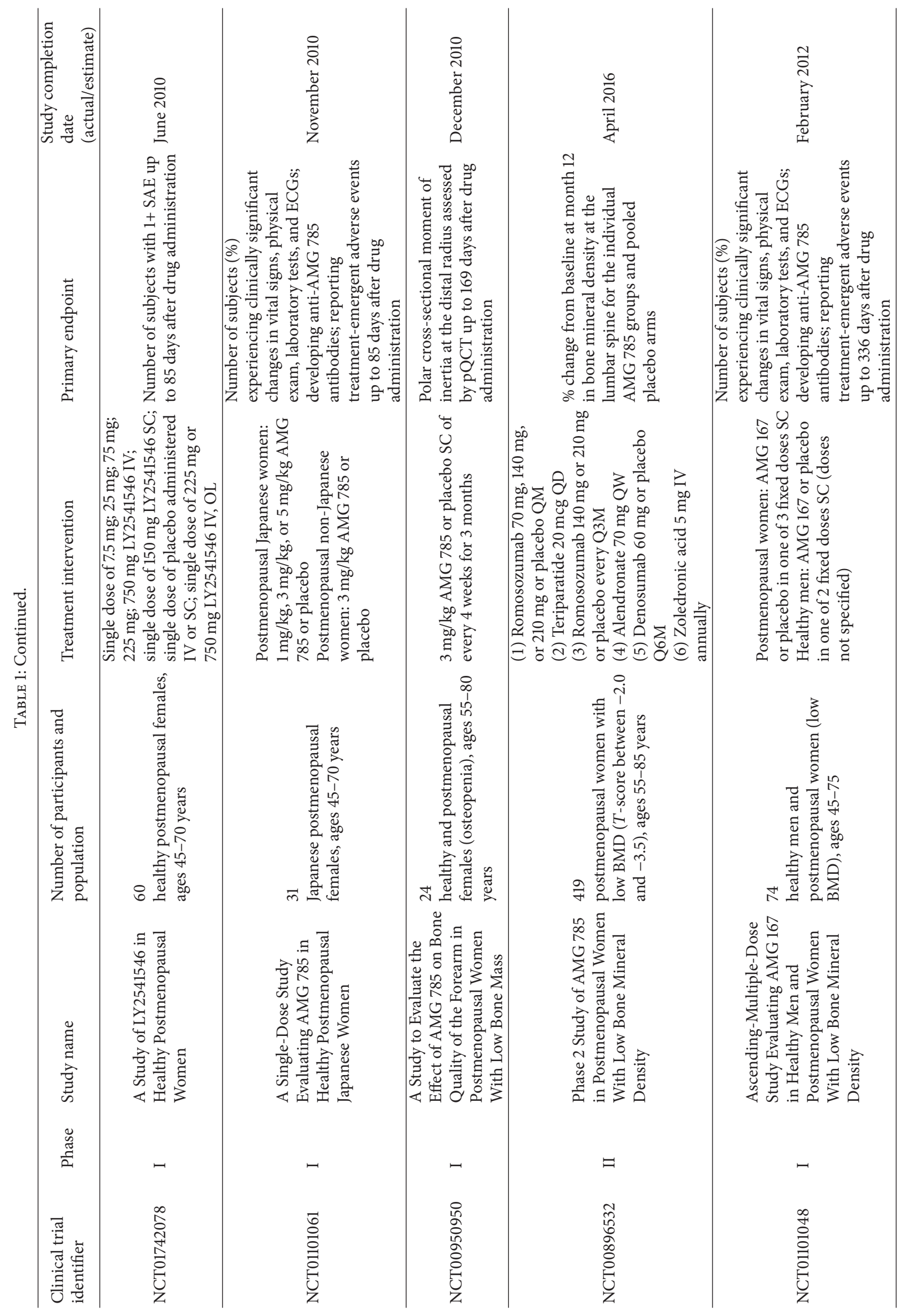




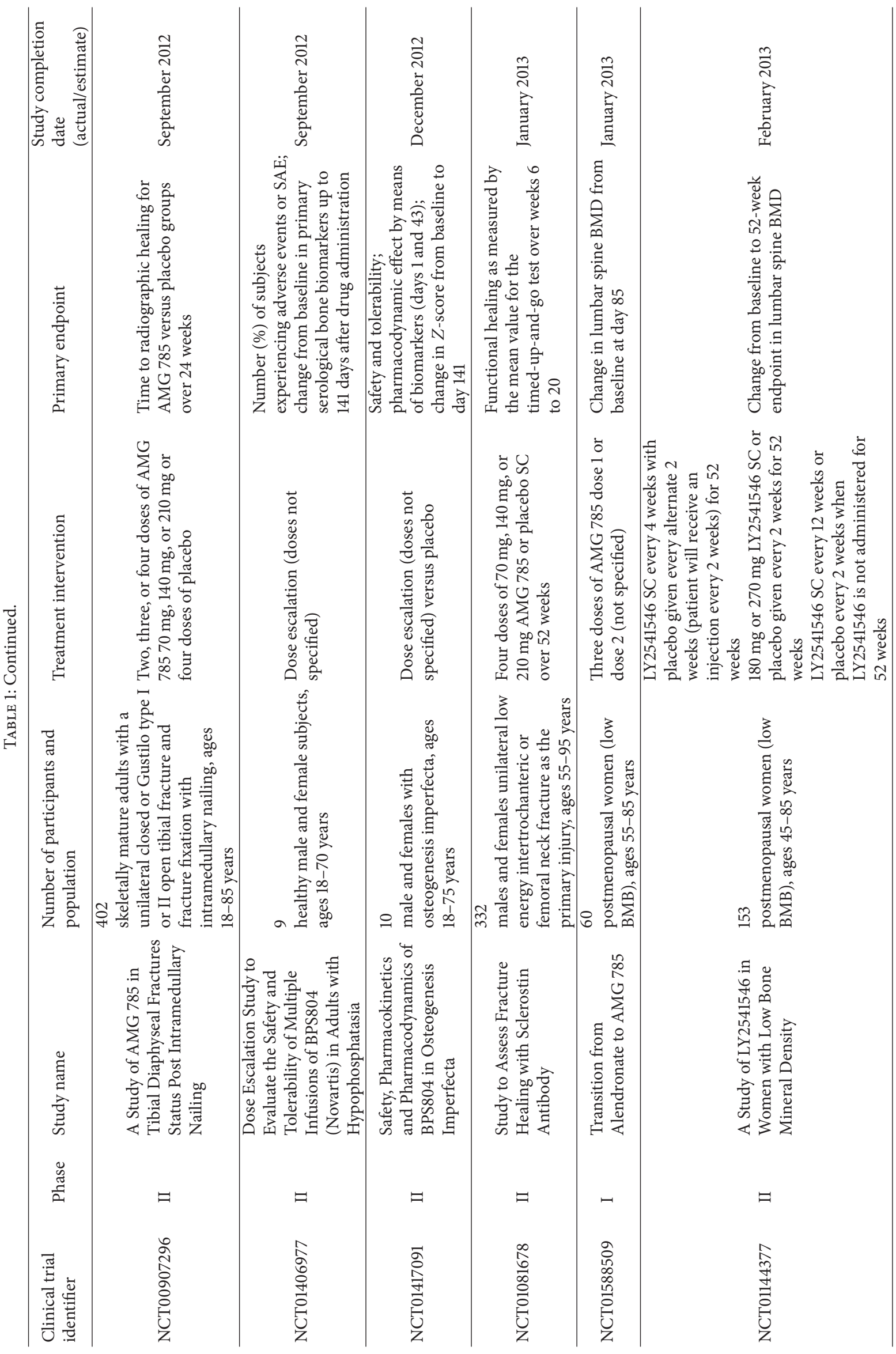




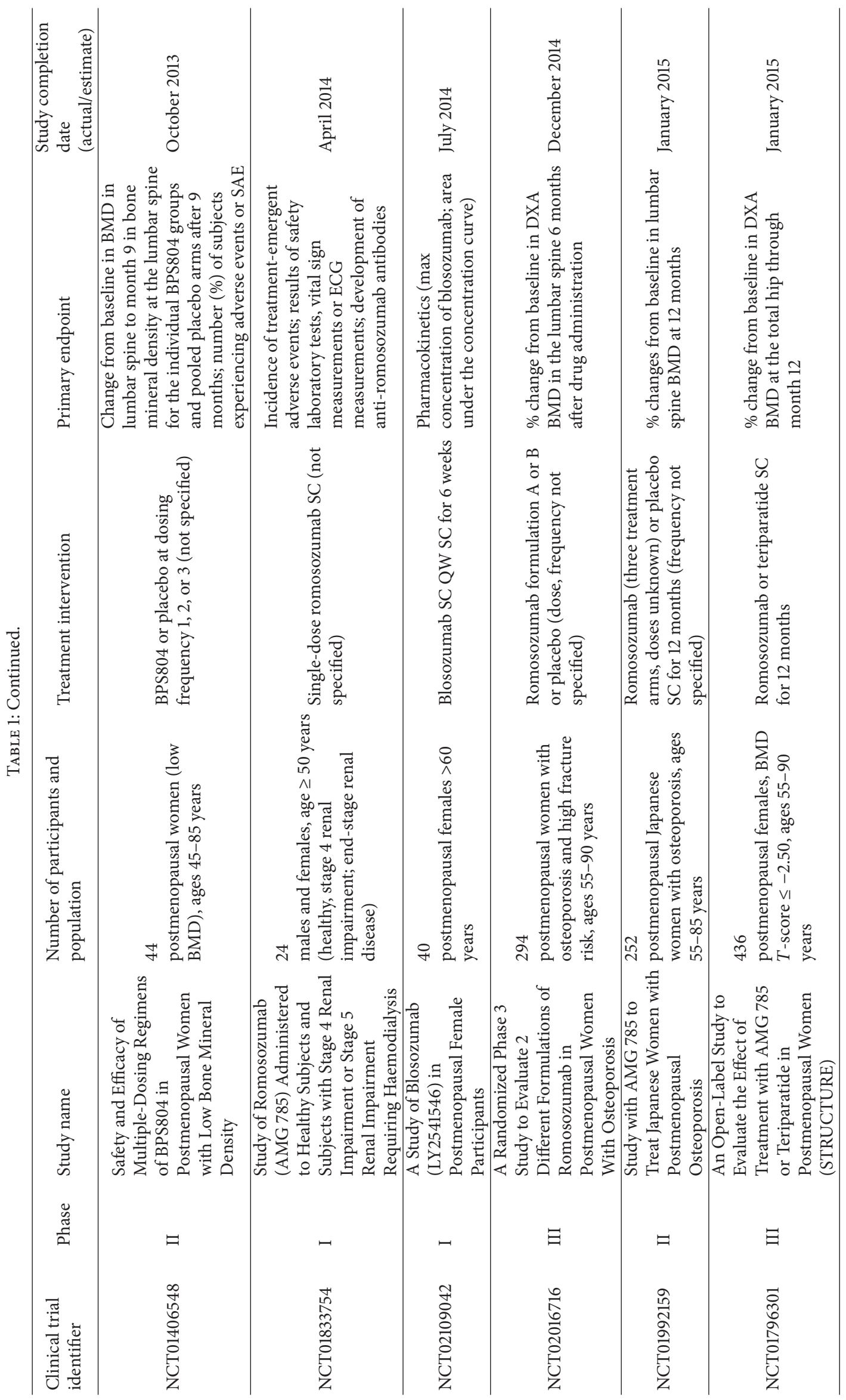




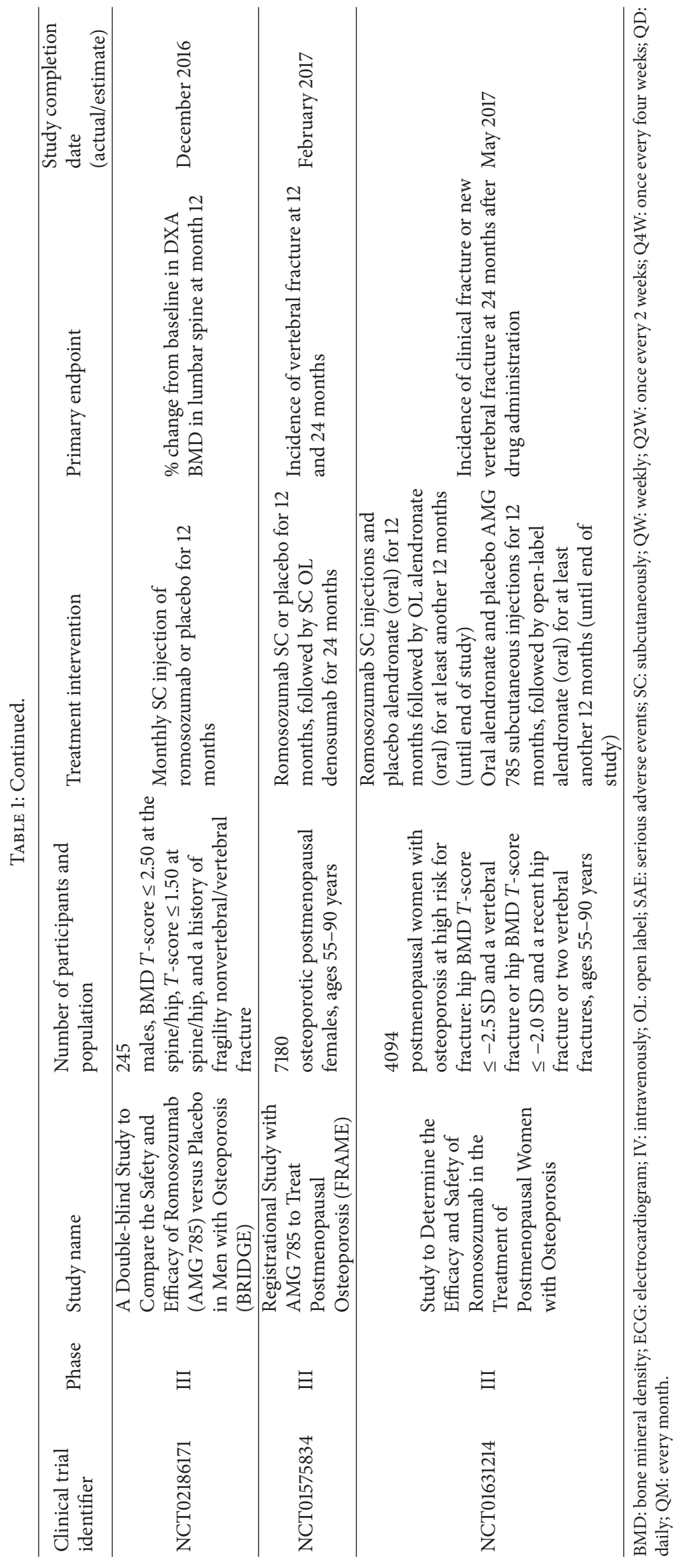




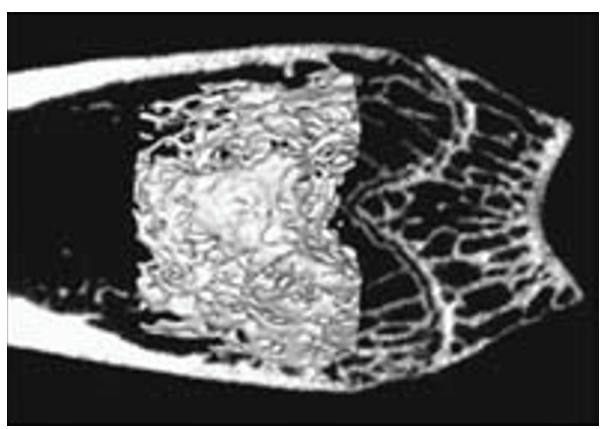

Region of analysis

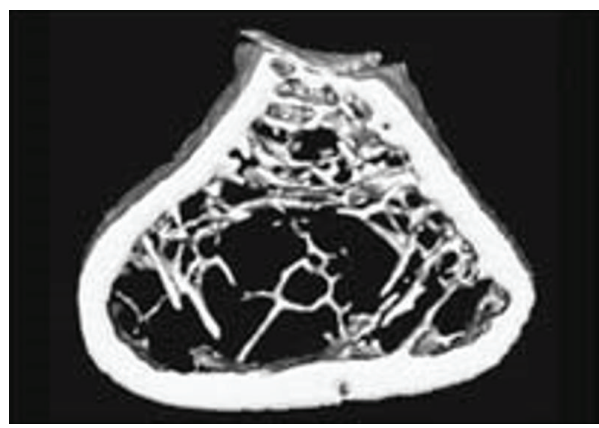

OVX + vehicle

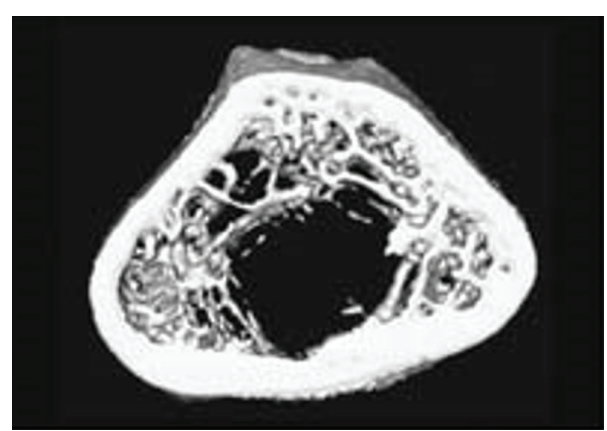

Sham

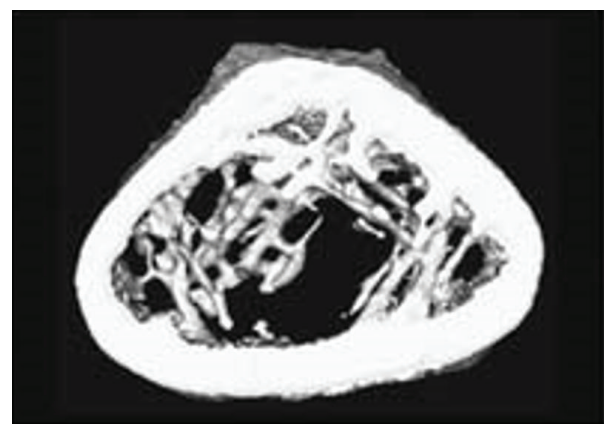

$\mathrm{OVX}+\mathrm{Scl}-\mathrm{AbII}$

(a)

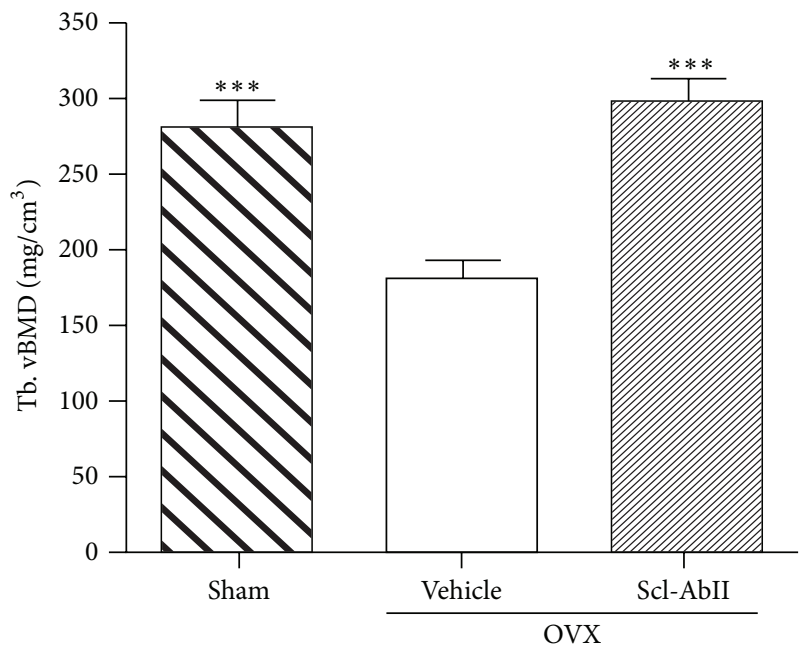

(b)

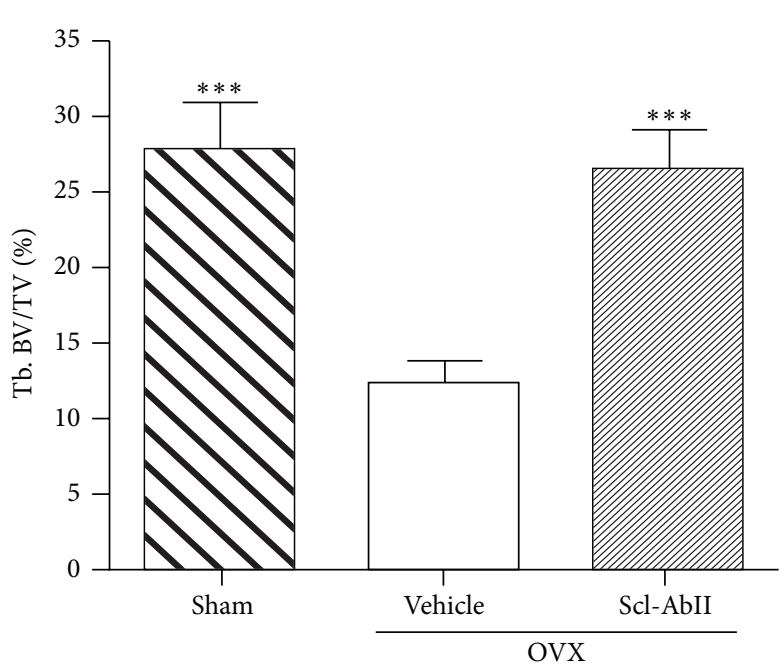

(c)

Figure 2: The first in vivo study to test anti-sclerostin antibodies for increasing bone mass involved a rodent model of postmenopausal osteoporosis. Ovariectomised (OVX) rats were treated with $25 \mathrm{mg} / \mathrm{kg}$ of sclerostin antibody twice weekly for 5 weeks while sham-operated controls (Sham) were treated with PBS. Treatment with sclerostin antibody reportedly restored trabecular bone volume and bone mineral density (BMD) to comparable levels observed in sham-operated animals. Trabecular volumetric BMD = Tb. vBMD; median trabecular bone volume $=\mathrm{Tb}$. BV/TV. ${ }^{* * *} p<0.001$ versus OVX + vehicle. Reproduced with permission from Li et al. [50].

was also significantly enhanced with maximum increases of $5.3 \%$ in the lumbar spine and $2.8 \%$ in the hip observed. Overall, treatment was generally well-tolerated with one serious adverse event being reported for a subject that received $10 \mathrm{mg} / \mathrm{kg}$ dose. The subject experienced nonspecific hepatitis which commenced 1 day after dosing but resolved after approximately 1 week. Of the 54 subjects receiving romosozumab $11 \%(6 / 54)$ tested positive for binding antiromosozumab antibodies in the highest-dose groups. Two of these subjects tested positive for neutralising antibodies (1 subject $10 \mathrm{mg} / \mathrm{kg}$ subcutaneous; 1 subject $5 \mathrm{mg} / \mathrm{kg}$ intravenous) [4]. Nonetheless, when placed in context with existing therapies, a single $10 \mathrm{mg} / \mathrm{kg}$ dose of Scl-mAb was found to produce $\mathrm{BMD}$ increases that were either equivalent or 
greater than that observed after 6 months of daily teriparatide treatment [54].

A subsequent double-blind, placebo-controlled, randomized, ascending-multiple-dose phase I trial (NCT01825785) was completed to assess the safety, tolerability, pharmacokinetics, and pharmacodynamics of romosozumab treatment for 12 weeks in 16 healthy men and 32 postmenopausal women with low bone mass [53]. Female subjects consisted of three groups. The first cohort received 6 doses of 1 or $2 \mathrm{mg} / \mathrm{kg}$ every 2 weeks (Q2W). The second group of participants received 3 doses of 2 or $3 \mathrm{mg} / \mathrm{kg}$ every four weeks (Q4W). The final group consisted of placebo treatment. The healthy male subjects received $1 \mathrm{mg} / \mathrm{kg}$ Q2W or $3 \mathrm{mg} / \mathrm{kg}$ Q4W or placebo. Romosozumab treatment in all groups was observed to increase PINP by $66-147 \%$ and lumbar bone mineral density by $4-7 \%$, associated with a concomitant $15-50 \%$ decrease in serum CTx. Total hip BMD also increased by approximately $3 \%$ in female subjects taking romosozumab. Bone formation marker levels (P1NP, osteocalcin, and BSAP) returned to baseline 4-8 weeks subsequent to the last romosozumab dose. With the exception of increased mild injection site reactions with romosozumab, adverse events appeared balanced between treatment and placebo groups. Similarly to the single-dose study, two subjects tested positive for neutralising antibodies which reportedly had no apparent effects on the primary study objectives [53].

McClung and colleagues have recently published results from a phase II, multicentre, international, randomized, placebo-controlled, parallel-group, eight-group study, where the efficacy and safety of romosozumab over a 12-month period was evaluated in 419 postmenopausal women (55-85 years of age; NCT00896532) [55]. Subjects were randomly assigned to three groups. These comprised either subcutaneous romosozumab monthly injections (dose of $70 \mathrm{mg}$, $140 \mathrm{mg}$, or $210 \mathrm{mg}$ ) or every 3 months (140 mg or $210 \mathrm{mg}$ ), subcutaneous placebo, or an open-label active comparatororal alendronate (70 mg weekly) or subcutaneous teriparatide (20 $\mu \mathrm{g}$ daily). The primary endpoint was the percentage change in $\mathrm{BMD}$ in the lumbar spine at 12 months compared to baseline. Secondary endpoints included the percentage change in BMD at other sites and changes in markers of bone turnover. After 12 months, the pooled romosozumab group had significantly higher BMD compared to the pooled placebo group irrespective of the frequency of dose (monthly/every 3 months) and dose concentration $(140 \mathrm{mg} / 210 \mathrm{mg})$. The largest gains were observed with the $210 \mathrm{mg}$ monthly dose of romosozumab. Here, mean increases (compared to baseline) of $11.3 \%, 4.1 \%$, and $3.7 \%$ in the lumbar spine, $4.1 \%$ total hip, and femoral neck were observed, respectively. Furthermore, the increases in BMD observed were significantly greater than the alendronate $(4.1 \%$ increase in BMD in the lumbar spine) and teriparatide groups (7.1\% increase in BMD in the lumbar spine).

Interestingly, serum levels of bone formation markers in this trial were reported to increase transiently. This contrasts the robust and reproducible increases in serum bone formation markers reported subsequent to romosozumab treatment in the iconic primate study [52]. McClung and colleagues observed that increases in serum bone formation markers occurred as soon as 1-week after administration with peak levels reported after 1 month. However, levels returned or fell below baseline values within 2-9 months (dependent on dose and marker evaluated). All romosozumab groups demonstrated a decrease from baseline in the level of CTx initially, with the largest median decrease evident after one week. Furthermore, subjects receiving monthly doses of romosozumab maintained levels of CTx below baseline after 12 months [55].

The incidence of serious adverse events in the pooled romosozumab group was $7 \%$ which was comparable to the placebo $(14 \%)$, alendronate $(8 \%)$, and teriparatide $(9 \%)$ groups. The $210 \mathrm{mg}$ (administered every 4 weeks) romosozumab group had $10 \%$ (5 subjects of 51) occurrence of serious adverse events. These included breast cancer, chronic obstructive pulmonary disease, noncardiac chest pain, wrist fracture, and begin renal oncocytoma. However, no serious adverse event was reported by more than 1 participant in any group and none were considered by the investigator to be treatment-related. As observed in the previous phase I studies, binding antibodies were identified in $20 \%$ of subjects receiving romosozumab, with $3 \%$ of these demonstrating in vitro neutralising activities. Despite their occurrence, there was no apparent effect on the occurrence of adverse events, pharmacokinetics, or pharmacodynamics [55]. Overall, given the significant and prompt increases in BMD subsequent to romosozumab treatment compared to alendronate and teriparatide, this study further supported the application of romosozumab in the treatment of osteoporosis.

More recently, this trial was extended to include an additional 12-month treatment period under similar study conditions as outlined above. Although only currently available in the abstract form, the increases in BMD in the lumbar spine and total hip observed during the first 12 months of treatment were further increased with the additional 12 -month romosozumab. The largest gains in BMD were again observed with the $210 \mathrm{mg}$ monthly dose (15.7\% lumbar spine BMD; $6.0 \%$ total hip BMD). This study was extended to include a one-year double-blind extension phase where eligible subjects (women receiving $210 \mathrm{mg}$ romosozumab dose) were rerandomized $1: 1$ within their original treatment group to placebo or denosumab $60 \mathrm{mg}$ once every six months $(n=260)$. Women receiving romosozumab $210 \mathrm{mg}$ monthly who then transitioned to treatment with denosumab (targets RANKL) after 12 months continued to accrue BMD at a rate similar to that observed during the second year of treatment with romosozumab. However, subjects that transitioned to placebo demonstrated a return in BMD towards pretreatment levels. Furthermore, in subjects where romosozumab treatment was discontinued after 2 years, a decrease in BMD towards baseline levels and the return of bone formation marker P1NP to pretreatment levels were observed [56].

Another ongoing study is assessing the impact of romosozumab treatment compared to teriparatide treatment on vertebral mass, thickness, and density. Although not published, initial results conveyed by Amgen suggest that treatment with romosozumab produced an increase in cortical thickness of $11.2 \%$ compared to $5.6 \%$ with teriparatide. Furthermore, romosozumab induced an increase of $22.2 \%$ 
in trabecular $\mathrm{BMD}$ compared to a $17.4 \%$ increase with teriparatide. In contrast, a reduction of $4.3 \%$ was reported in the placebo group (NCT01796301). A phase I trial assessing changes in baseline lumbar spine BMD after the transition from alendronate to romosozumab for 3 months has recently been completed with results pending (NCT01588509). It would certainly appear that romosozumab has great promise in the treatment of osteoporosis and based on these results it would appear that market approval may be foreseeable. Less clear at the moment, however, are efficacy issues regarding long-term treatment (i.e., in excess of 2 years as a decrease to baseline is observed after discontinuation) and safety concerns relating to potential long-term treatment schedules. For the moment, at least, it would appear that romosozumab would be highly beneficial in the short-term and is compatible with antiresorptive therapies.

4.2. Blosozumab. Two phase I trials of blosozumab (Eli Lilly) were undertaken. The first study included a single-dose, dose escalation study (7.5 to $750 \mathrm{mg}$ i.v.; NCT01742078). This was followed by a multicentre safety and tolerability study of multiple-dose administration (Q2W, Q4W; NCT01742091) [57]. Participants were otherwise healthy postmenopausal women ranging in age within $45-70$ years of age (singledose) and 45-80 years of age (multidose). Both studies were subject and investigator blinded and placebo-controlled. Overall, the treatment was generally well-tolerated. However, antibodies to the drug were discovered upon screening $(23 \%$ of patients in the single-dose study; $36 \%$ in the multidose study). Nonetheless, titres were low and their occurrence did not appear to impact pharmacodynamics. Furthermore, neutralising antibodies were not detected. Dose-dependent increases in drug serum levels and bone formation markers P1NP and BAP and osteocalcin along with a decrease in CTx were recorded. Treatment also resulted in increased BMD in the lumbar spine with a maximum increase above baseline of $3.41 \%$ after a single dose and $7.71 \%$ after multiple doses after 85 days (compared to $5.3 \%$ and $7.2 \%$, resp., for single and multiple-dose administration of AMG 785).

These positive clinical results were followed in a more recently randomized, double-blind, placebo-controlled multicentre phase 2 clinical trial of blosozumab comprising 120 postmenopausal women (45-85 years) with low bone mineral density (lumbar spine BMD T-score of -2.0 to -3.5; NCT01144377). The study encompassed a 1-year treatment period with a 3-month follow-up period once treatment ended. The primary objective was to evaluate the doseresponse of $180 \mathrm{mg}$ (every 2 or 4 weeks) to $210 \mathrm{mg}$ (Q2W) (compared to $70 \mathrm{mg}-210 \mathrm{mg}$ in the phase two trial of romosozumab) of blosozumab on lumbar spine BMD [21].

In terms of efficacy, mean increases in BMD in the lumbar spine were statistically significant for all treatment groups. A maximum increase in the lumbar BMD of $17.7 \%$ above baseline in the group receiving $270 \mathrm{mg}$ every two weeks was reported. In the 3-month follow-up study, the BMD in all dosage groups declined when blosozumab treatment ceased. Levels of P1NP peaked at week 4 and remained significantly above baseline for 24 weeks and returning towards baseline levels by the end of the study. Levels of osteocalcin and bone alkaline phosphatase also increased with blosozumab treatment and returned towards baseline by the end of the study. The bone resorption marker CTx levels decreased to less than placebo within two weeks and were similar to placebo at 12 weeks and reduced compared to placebo at 52 weeks [21].

Mild injection site reactions were reported by up to $40 \%$ of patients receiving blosozumab; otherwise, adverse events were similar between treatment and placebo groups. Four women taking blosozumab were diagnosed with breast cancer within 3 months to 1 year of the beginning of the trial; however, examination suggested these were likely to be preexisting tumours. Anti-blosozumab antibodies were found in $35 \%$ of those treated with one patient developing neutralising antibodies that resulted in greatly reduced efficacy from treatment [21].

A one-year follow-up study assessing the effects of discontinuing blosozumab treatment in the phase II patient cohort has recently been published [58]. No serious adverse effects after discontinuation of the treatment were observed. With the discontinuation of treatment, a decline in BMD in both the femoral neck and the lumbar spine was observed in all blosozumab treatment groups, which continued through the 1-year follow-up period. However, these remained significantly higher than placebo groups. Moreover, serum biochemical formation and resorption markers did not differ significantly between previously treated blosozumab and placebo groups. While in the initial phase II trial approximately $35 \%$ of blosozumab treated patients presented with antitreatment antibodies, these were seen to decline with discontinuation of the treatment [58].

Overall, the results from the phase two trial were encouraging. While no serious safety issues were raised, John Lechleiter the Chairman of the Board, President, and Chief Executive Officer of Eli Lilly explained that phase III trials proposed for 2014 were delayed due to higher than desirable levels of injection site reactions. This led to the reassessment of the formulation used in their phase II trials before moving forward. The occurrence of injection site reactions appears to be a common occurrence subsequent to antisclerostin treatment for all humanized Scl-mAb tested and is currently under investigation by both Amgen and Eli Lilly.

4.3. BPS804. Three BPS804 phase II trials to treat postmenopausal women with low BMD (NCT01406548), osteogenesis imperfecta (NCT01417091), and hypophosphatasia (NCT01406977) have been completed. To date, no data has been published and there is no information available in relation to plans for phase III trials. An additional phase II trial to investigate the safety and tolerability of BPS804 in patients with late-stage chronic kidney disease (NCT01806610) has since been withdrawn prior to initiation of patient enrolment. Not surprisingly, therefore, BPS804 does not currently feature in the clinical pipeline reports for 2014-2018 published by Novartis [59]. Nevertheless, the company's interest in fundamental research pertaining to the mechanistic actions of sclerostin in bone repair persists [60-62].

4.4. Combined Treatments. The combined therapy approach of $\mathrm{Scl}-\mathrm{mAb}$ antibodies followed by ongoing use of antiresorptive 
drugs to maintain enhanced bone formation is another emerging area of investigation. The use of alendronate in relation to pretreatment and cotreatment with antisclerostin therapy has been assessed in animal studies with encouraging results [63]. This stands in contrast to the currently approved anabolic treatment with human parathyroid hormone (PTH). In this case, it was hypothesised that cotreatment with alendronate would enhance the anabolic qualities of PTH. However, the combined treatment was actually found to have a reduced anabolic effect in clinical studies $[64,65]$.

The cotreatment of anti-tumour necrosis factor (antiTNF) along with anti-sclerostin antibodies in human TNF transgenic mice has recently been shown to be more effective than either treatment administered alone. Specifically, cotreatment of mice with anti-TNF and Scl-mAb was found to be effective in repairing cortical lesions, cartilage destruction, and preventing proteoglycan loss. These results were an improvement compared to anti-TNF or Scl-mAb alone which appeared to prevent further disease progression but did not support tissue repair. For instance, in this model, the combination of anti-TNF and Scl-mAb treatment was observed to prevent cortical and trabecular bone loss (an increase of $34 \%$ compared to baseline) and restored vertebral bone to levels observed in nonarthritic wild-type mice. The combination of anti-TNF and Scl-mAb significantly decreased arthritic bone erosion compared to baseline as well as reducing osteoclast number. Interestingly, compared to baseline levels, Scl-mAb alone or in combination with anti-TNF significantly increased cartilage thickness, area, and proteoglycan content. Interestingly, the largest increases were observed in the cotreatment groups [43].

This combination of anti-inflammatory treatment with bone enhancing antibodies could also have potential to aid the treatment of decreased BMD due to colitis. The option of alternating anabolic and antiresorptive therapies has also been the focus of several recent clinical trials relating to romosozumab treatment and is highlighted in Section 4.1.

\section{Emerging Alternative Clinical Applications for Sclerostin mAb Therapy}

5.1. Fracture Healing. The efficacy of anti-sclerostin antibody therapy in the treatment of fragility fractures has been tested in rodent and nonhuman primate preclinical models [66-69]. More recently, two phase II clinical trials (NCT00907296) (NCT01081678) have also investigated this application. In all rodent experimental models (rat diaphyseal defect, rat closed mid-diaphyseal femoral fracture, rat femoral osteotomy, and murine femoral osteotomy), subcutaneous administration of $25 \mathrm{mg} / \mathrm{kg}$ of Scl-mAb twice weekly for varying time courses confirmed enhanced bone healing with significant increases in bone formation, mass, and strength [66-69]. Furthermore, Cui and colleagues report that the size of the repair callus in Scl-mAb treated mice with osteotomies was increased as early as 2 weeks after treatment compared to controls. The Scl-mAb treated group was associated with a faster fracture union by week 6 and significantly higher maximal loading capacity [66]. Similarly, Suen and colleagues report an increase in fracture callus size of $23-30 \%$ at 3,6 , and
9 weeks after Scl-mAb administration compared to vehicle controls. Histologically more bony tissue and less cartilage tissue were observed in fracture calluses across all time points in the Scl-mAb treated groups. Moreover, the proportion of mature callus tissue was significantly greater with the Scl$\mathrm{mAb}$ treatment at weeks 6 and 9 . This was reflected by the significant increases in total bone volume (26-33\%) and highdensity bone volume (38-42\%) compared to vehicle groups. This was also associated with more rapid progression of fracture repair supported histologically. The Scl-mAb treatment also resulted in faster mineral deposition compared with vehicle controls. Furthermore, Scl-mAb increased the rate of new bone formation in both the total callus (41\%) and the periosteal callus subregion (42\%) at 9 weeks. These positive effects of Scl-mAb treatment also translated to mechanical outcomes. Specifically, a significantly higher load in Scl-mAb treated groups was reported at weeks 6 (98\%) and 9 (53\%) after fracture compared to controls [67]. Similarly, Scl-mAb treatment resulted in significant increases in callus stiffness and energy to failure. The positive impact of Scl-mAb treatment in fracture repair setting is also reflected elsewhere [70].

More recently, Yee and colleagues have demonstrated enhanced bone formation using Scl-mAb in an early onset Type I diabetic mouse fracture model. Here the authors report that $\mathrm{Scl}-\mathrm{mAb}$ treatment rescued impaired osteogenesis and marrow adiposity that is associated with the diabetic phenotype. Moreover, in uninjured bone, the positive effect of $\mathrm{Scl}-\mathrm{mAb}$ on bone formation persisted for up to 3 weeks after discontinuation of the biweekly $(25 \mathrm{mg} / \mathrm{kg})$ treatment [71]. Despite the promise of Scl-mAb treatment in fracture repair, the reparative effect does not appear to reproducibly extend to nonunion fractures [72, 73]. For example, Alaee et al. have recently demonstrated that treatment with Scl-mAb can enhance bone repair in the surrounding bone of a rat femoral critical-sized bone defect but does not possess osteoinductive activity to heal it [73]. Thus, it seems that Scl-mAb is effective in enhancing bone formation of preexisting and regenerating bone tissue but does not appear to be an osteoinductive agent. This appears to be supported by a recent study that has shown that endochondral bone formation persists in fractures lacking sclerostin (SOST -/- mice) while fibrocartilage callus removal was enhanced. The resultant bony calluses displayed increased bone fraction and strength [62].

Ominsky et al. examined Scl-mAb treatment for the repair of bilateral fibular osteotomies in cynomolgus monkeys [68]. Treatment consisted of $30 \mathrm{mg} / \mathrm{kg}$ of anti-sclerostin antibody twice weekly for 10 weeks. The therapy led to an increase in serum levels of the bone formation markers osteocalcin and procollagen $1 \mathrm{~N}$-terminal propeptide (P1NP) and an associated increase in bone formation. After just 7 weeks, fractures in the vehicle and $\mathrm{Scl}-\mathrm{mAb}$ groups healed to $27 \%$ and $48 \%$ of the mean peak load of the intact contralateral femurs in the vehicle group. In addition, Scl$\mathrm{mAb}$ administration significantly improved the rate by which the majority of intact strength was achieved compared to vehicle-treated groups. The rate to union was also markedly improved with Scl-mAb treatment. Specifically, 9/10 in the latter group achieved union compared to $4 / 9$ fractures in the vehicle-treated group. Histologically, there was a significant 
increase in bone formation within the fracture area in Scl$\mathrm{mAb}$ treated groups. This was associated with reductions in the persistence of a cartilaginous callus within this group. The smaller fracture gaps observed within the Scl-mAb groups also displayed less fibrovascular tissue compared to vehicletreated groups, although this difference was not statistically significant. At the fracture site, Scl-mAb treatment resulted in a $27 \%$ increase in mature bone callus formation associated with a $30 \%$ increase in bone mineral content. Furthermore, Scl-mAb produced a $48 \%$ greater mean torsional stiffness and $32 \%$ greater peak torque compared to vehicle controls [68].

Both phase II trials assessing Scl-mAb in fracture repair (NCT00907296 and NCT01081678) directed by Amgen have submitted requests in May 2013 and January 2014, respectively, to delay publication of the trial results. At the time of writing, neither blosozumab (Eli Lilly) nor BPS-804 (Novartis) appears to be undergoing investigation at clinical trial. Therefore, to date, the efficacy of Scl-mAb therapy in the application of fracture repair has not been established in human trials. Furthermore, a press release early in 2013 confirmed that Amgen would not be pursuing Scl-mAb treatment for fracture healing into phase III trials [74]. Sclerostin levels are greatly increased in the early stages of fracture healing which may add to the complexity of providing effective neutralisation to enhance fracture repair [75]. Fracture repair and related aftercare currently account for a staggering 95\% [12] of the cost of all osteoporosis treatments. The lack of Scl-mAb therapy in this area will see patients remain restricted to the prophylactic treatment options to reduce fracture risk for the foreseeable future.

5.2. Implant Fixation. The secure fixation of bone implants presents another complication in the treatment of osteoporosis. High failure rates are reported for total hip replacement due to the weakness of the surrounding bone. The anchorage of screws and pins used in fracture repair can also be compromised due to poor bone quality $[10,11]$. Preclinical studies have demonstrated efficacy in significantly enhancing bone fraction volume adjacent to the implant subsequent to Scl-mAb administration. Treatment has also been shown to increase peak pull-out forces [76, 77]. Moreover, screws inserted postmortem also showed increased pull-out force in the treated group. This would appear to imply a general rather than injury specific response to antibody treatment [76]. Moreover, Scl-mAb administration has demonstrated promise in preventing periprosthetic osteolysis and aseptic loosening in a preclinical study [78]. More recently, in a severe model of osteoporosis (approximately 78\% trabecular bone loss at the time of implantation) systemic Scl-mAb administration has also proven effective in stimulating osseointegration via enhanced bone formation. Here, the authors report improved trabecular bone volume and architecture as well as decreased bone resorption subsequent to Scl-mAb treatment [79].

5.3. Treatment of Other Diseases Impacting Bone. In addition to the treatment of osteoporosis, antisclerostin therapy has been explored as a treatment for other conditions causing reduced bone mineral density. Preclinical animal studies encompassing periodontitis, colitis, rheumatoid arthritis, osteoarthritis, bone health complications of diabetes mellitus, chronic kidney disease, and osteogenesis imperfecta (OI) have all been encouraging [80-85]. However, there appears to be some contradiction between studies possibly resulting from the different animal models used or the disease stage in which Scl-mAb were administered. For instance, treatment of severe OI with Scl-mAb had limited success in enhancing trabecular bone volume and cortical thickness in growing mice ( 4 weeks). In contrast, treatment failed to have any significant impact in adult OI mice (20 weeks). Furthermore, neither growing nor adult OI mice displayed treatmentassociated alterations in bone remodelling serum markers compared to wild-type controls [86]. In contrast, moderately severe $\mathrm{OI}$ in the $\mathrm{Brtl} /+$ mouse model of OI responded positively to Scl-mAb treatment. Here, treatment resulted in stimulated osteoblast-mediated bone formation leading to increases in bone mass and reduced long-bone fragility $[83,86]$. Bisphosphonates are currently used in the treatment of OI and have been shown to lower the risk of fracture in some cases $[87,88]$. While generally well-tolerated, there are, however, unresolved issues relating to the use of bisphosphonates in OI. These include issues relating to long-term retention, clinical reproducibility of lowering fracture risk, functional benefit, and potential childhood bisphosphonate toxicity [89-92]. Moreover, other anabolic treatments such as growth hormone and teriparatide have provided mixed outcomes. For instance, prolonged use of teriparatide may be potentially associated with the development of osteosarcoma $[93,94]$. Given these issues and the observed anabolic effect of $\mathrm{Scl}-\mathrm{mAb}$ in osteoporosis and fracture repair, it is speculated that this therapy may be beneficial in the treatment of OI. Specifically, it is thought that Scl-mAb treatment may benefit paediatric OI patients in particular by increasing bone mass and mechanical strength and reducing the risk of fracture. A 2-week treatment course of Scl-mAb was found to produce increases in bone mass and mechanical strength in the wellestablished Brtl/+ mouse model of moderately severe Type IV OI. However, more extensive studies are required to fully establish the potential of Scl-mAb in OI [83], Moreover, given that it is likely that Scl-mAb therapy will need to be administered as an adjunctive therapy (see Section 7), the long-term benefits in OI need to be addressed.

Recently, Chen et al. combined anti-TNF $t$ with Scl$\mathrm{mAb}$ treatment in a mouse model of rheumatoid arthritis. The combination therapy was found to contribute to repair of damaged articular cartilage and eroded bone, whereas either treatment alone only prevented progression of disease symptoms [81]. Chondrocytes reportedly secrete sclerostin. However, a recent study has suggested that pharmacological inhibition of sclerostin does not impact articular cartilage remodelling; therefore, an explanation for the positive outcomes described above is yet to be fully elucidated [81, 95].

Periodontitis, a destructive disease of the tooth-supporting structures ultimately resulting in tooth loss, is another area which may potentially benefit from Scl-mAb therapy to repair large osseous defects. Recent evidence suggests that sclerostin (as well as DKK-1) is significantly increased in the gingival tissue and the serum of chronic periodontitis 
patients. These findings suggest a possible molecular link between sclerostin and periodontal disease [96]. Using a variety of methods including lineage tracing and knock-out models, it has recently been shown that activation of the Wnt-canonical pathway via Scl-mAb treatment results in cementum and alveolar bone regeneration [85, 97, 98]. Taut and colleagues recently investigated the effect of administering $25 \mathrm{mg} / \mathrm{kg} \mathrm{Scl-mAb}$ administration delivered twice weekly for a therapeutic duration of 3 and 6 weeks in an experimental periodontal rat model. Notably, after 6 weeks, the Scl-mAb treated group displayed reversed ligature-induced bone loss. This outcome was also associated with a significant increase in bone volume and tissue mineral density compared to vehicle-treated controls. Interestingly, local administration of Scl-mAb displayed limited effect on volumetric alveolar bone healing. Alveolar bone loss was, however, significantly improved after 6 but not 3 weeks subsequent to systemic Scl$\mathrm{mAb}$ treatment compared to vehicle-treated groups. Bone densitometry scanning was also performed on femora to assess off-site skeletal responses to Scl-mAb administration. The femora of all treatment groups demonstrated significant increases in bone mineral density at both 3-and 6-week time points. Bone formation markers were also increased after Scl-mAb administration which translated to the restoration of lost bone microarchitecture, volume, and density to levels comparable to intact control after 6 weeks. Interestingly, the application of Scl-mAb as a preventative measure to alveolar bone loss (administered when sutures were initially placed and while at the site of defect) only resulted in a slight and nonsignificant increase in bone mass after 2 and 4 weeks. These findings highlight the suitability of Scl-mAb as an adjunctive therapy in this context [99].

Chen and coworkers also studied the effect of administering $25 \mathrm{mg} / \mathrm{kg}$ vehicle or Scl-mAb subcutaneously twice weekly for 6 weeks in ovariectomised rats with experimental periodontitis (ligature). The authors noted that the administration of Scl-mAb leads to significant increases in the bone mineral apposition rate in ovx-rats compared to controls. The authors attribute this finding to increased bone formation and decreased bone resorption evident from significant increases in osteocalcin and osteoprotegerin and serum tartrate-resistant acid phosphatase and CTx, respectively [100]. It is worth considering that current osteoconductive agents, such as bone substitutes used in this application, are clinically unpredictable in their consistency to form bone [101]. Consequently, in an effort to recapitulate the complex biological events involved in wound healing and repair, a multiphasic approach is becoming necessary [102]. As noted previously, current evidence would suggest that Scl-mAb possesses osteoconductive rather than osteoinductive properties $[62,73]$. Therefore, it remains to be seen if (a) Scl-mAb is superior to current available osteoconductive agents such as commercial bone substitutes/grafts at producing more clinically consistent bone regeneration and (b) if Scl-mAb treatment, either systemically applied or locally administered via a multiphase scaffold or current commercial grafts, could provide a more robust outcome of targeted bone repair.

Growing evidence suggests that circulating Wnt signalling inhibitors such as DKK-1 and sclerostin may crucially contribute to the pathogenesis of chronic kidney diseaseassociated bone mineral disorder (CKD-MBD). Consequently, these have been explored as therapeutic candidates. Serum sclerostin levels have been shown to increase with CKD-MBD progression and may be potentially linked with cardiovascular events observed in this patient population $[103,104]$. In addition to its prominent role in bone remodelling, the Wnt pathway and several of its inhibitors including sclerostin are increasingly associated with the occurrence of extraosseous mineralisation. This occurrence is similar to that observed in cardiovascular calcification and calciphylaxis, a rare life-threatening condition which manifests predominately in patients with CKD or end-stage renal disease $[105,106]$. The biological similarities of vascular calcification and bone formation are gaining increased attention and it is believed that BMP-2, in particular, may be a vital link. Specifically, BMP-2 function can be inhibited by active matrix Gla protein (MGP), the activator of which is vitamin $\mathrm{K}$ dependent [107]. Moreover, vitamin K antagonist usage has been implicated as an independent risk factor for the development of calciphylaxis [108]. Sclerostin also antagonizes the effect of BMP-2 indirectly via inhibition of the Wnt/ $\beta$-catenin signalling pathway. This may help explain the biological link between circulating serum sclerostin levels and its link to cardiovascular events observed in recent studies [109]. Nevertheless, the potential of sclerostin as a biomarker in this context requires additional confirmation. Preliminary evidence in a cross-sectional multislice computed tomography scanning study of 67 chronic haemodialysis patients suggests that increased sclerostin expression was colocalised at the sites of calcifying aortic heart valve disease [105]. Others have implicated increased serum sclerostin levels in CKD patients to be statistically correlated with inflammation, vascular lesions, uremia, and potentially mortality [110]. In contrast, others have found a contradictory correlation whereby higher levels of serum sclerostin were associated with improved survival in prevalent haemodialysis patients [111]. Moreover, in a recent prospective study of 673 incident dialysis patients, increased serum levels of sclerostin were associated with lower short-term (up to 18 months) cardiovascular (hazard ratio 0.29 ; 95\% CI 0.13-0.62) and allcause mortality (hazard ration 0.39 ; $95 \%$ CI $0.22-0.68$ ). This trend became less significant over a 4 -year follow-up period [112]. At this point, there is no clinical data to underline the therapeutic efficacy of Scl-mAb in CKD-MBD. However, recent preclinical animal studies have reported enhanced bone volume and mineralisation with Scl-mAb treatment specifically when PTH levels are low $[113,114]$.

\section{Ongoing Developments: Antibody Fragments and Single Chain Antibodies}

Good tolerability has generally been reported subsequent to Scl-mAb administration. However, the development of neutralising antibodies to antisclerostin treatment has been reported including one case which seriously reduced the efficacy of treatment $[4,21,53,55]$. Moreover, the potential immunogenicity of full-size monoclonal antibodies has been raised as a general safety concern [95]. Ultimately, 
ongoing phase III trials of full-size sclerostin neutralising antibodies will determine if immunogenicity is sufficient to cause concern. Nevertheless, while isolated, these events have contributed to the ongoing development of smaller antibody fragments $[95,115,116]$. For instance, Roudier et al. have shown the sclerostin-neutralising activity of sclerostin antibody fragment (Scl-Fab; MW $48 \mathrm{KD}$ ) to be as effective as that of the parent Scl-mAb IgG in the rat medial meniscus tear model of osteoarthritis. Moreover, the sclerostin single chain fragments (Scl-scFv; $35 \mathrm{KD}$ ) were observed to increase bone density, enhance bone formation, and improve bone microstructure in a rat preclinical model of osteoporosis. However, while Scl-scFv demonstrated high specificity and affinity, the authors note it had lower stability than the fullsized antibody [116].

\section{Clinical Considerations}

The route to market approval for any novel therapeutic agent is never easy and in the case of antisclerostin treatment the stakes are incredibly high. Years of research stand in the balance along with an estimated $\$ 99$ million in global sales annually if market approval is secured [117]. While encouraging results from both preclinical and clinical studies have grabbed attention, a relatively small number of clinical safety and efficacy considerations have emerged. One serious adverse event has been reported in the first clinical trial of AMG785 (NCT01059435). Here, one subject developed severe nonspecific hepatitis which began one day after receiving $10 \mathrm{mg} / \mathrm{kg}$ of AMG785 (romosozumab). The hepatitis was resolved after 26 days, and additional cases of hepatitis have not been reported in subsequent trials to date [4].

As previously mentioned, the study of sclerosteosis and van Buchem disease uncovered the role of sclerostin in the modulation of the Wnt/ $\beta$-catenin dependent pathway. As a precaution, therefore, prominent health issues relating to both conditions were investigated. While heterozygous carriers of sclerosteosis have higher than normal BMD, they are otherwise considered clinically normal [118]. However, bone overgrowth in the skull that constrains the cranial nerves and induces facial palsy can occur. Deafness can also arise due to impaired movement of middle ear ossicles [14]. A phase II clinical trial of blosozumab tested both clinical manifestations. There was no indication of a disproportionate increase in the bone mineral content of the skull. Results from brainstem auditory evoked potential tests to determine any negative impact on nerve function and hearing were also considered unremarkable by a blinded clinician [21].

The Wnt/ $\beta$-catenin signalling is involved in a wide variety of developmental and adult tissue processes. Unsurprisingly, therefore, dysregulation of this integral pathway is associated with a multitude of diseases including cancer, fibrosis, and neurodegeneration $[119,120]$. A commercial therapeutic specifically targeting the Wnt-pathway is not currently available. With market approval of Scl-mAb fast approaching, it is worth considering potential clinical implications [121]. Recent work has shown that constitutive activation of $\beta$-catenin can potentially negatively impact bone. Jia and colleagues constitutively activated $\beta$-catenin using Catnb+/lox(exon 3) mice which were crossed with mice expressing a tamoxifen-inducible procollagen I CreER promoter. They demonstrate that constitutively activated $\beta$-catenin resulted in an excessive bone volume within the vertebral column in both early postnatal (3 days) and mature tissue (up to 7 months postnatally) [122]. However, the authors noted that early stabilisation of $\beta$-catenin essentially slowed linear bone growth within the vertebrae by retarding growth plate maturation. This resulted in shorter stature mice. Moreover, histologically the excessive newly formed bone appeared immature and occurred primarily adjacent to the growth plate. In contrast, late-stage $\beta$-catenin stabilisation appeared to not affect bone maturity or distribution [122].

More recently, it has also been shown that timely suppression of the Wnt/ $\beta$-catenin pathway is required for osteocytic differentiation to occur adequately. In this study, $\beta$ catenin was constitutively activated in osteocytes by crossing Catnb+/lox(exon 3) mice with dentin matrix protein 1Cre transgenic mice [123]. Interestingly, stabilisation of $\beta$ catenin in osteocytes was observed to substantially increase cancellous bone mass. However, the activation was noted to have severe adverse effects on bone strength and bone growth. Specifically, impaired mineralisation resulted in thinner and more porous cortical bone. Similar to Jia et al. [122] mice were shorter and presented with impaired linear growth of the long bones [123]. The integral role of $\mathrm{Wnt} / \beta$-catenin signalling in development and disease, as well as the effects described in recent studies, should certainly be seriously considered prior to the adoption of an application involving its therapeutic interference.

Direct comparisons between humanized Scl-mAb are currently unavailable. However, a curious difference between a recent phase II blosozumab study and a prominent primate study assessing romosozumab is of potential interest $[21,52]$. Clearly, comparison of a year-long human clinical trial and a 2-month long primate study assessing structurally different humanized forms of Scl-mAb is anecdotal. Nevertheless, in the absence of an explanation, it is worth briefly discussing. In the human clinical trial using blosozumab, P1NP levels increased promptly during the first 4 weeks of treatment. Concurrently, the serum concentration of the bone resorption marker CTx rapidly decreased to a concentration below that noted for the placebo group within the first 2 weeks of treatment. By the end of the study, P1NP levels were comparable to pretreatment levels while CTx remained reduced [21].

The authors suggest that the return of P1NP towards baseline levels in the later stage of treatment (after week 25) may be a result of the initial increase in bone formation reducing stresses and strains on the skeletal system. Consequently, the positive signal to induce bone formation is dampened. However, this was not apparent in primate studies (Figure 3) [52]. Moreover, signalling molecules such as DKK1 may be actively involved by reducing bone formation via negative counterregulation [124]. Importantly, the observations that blosozumab increases bone formation, decreases bone resorption, and increases spine and total hip BMD are consistent with the recently published romosozumab phase II trial comprising over 400 subjects [55]. The clinical implications, if any, in the discrepancy of serum P1NP levels 


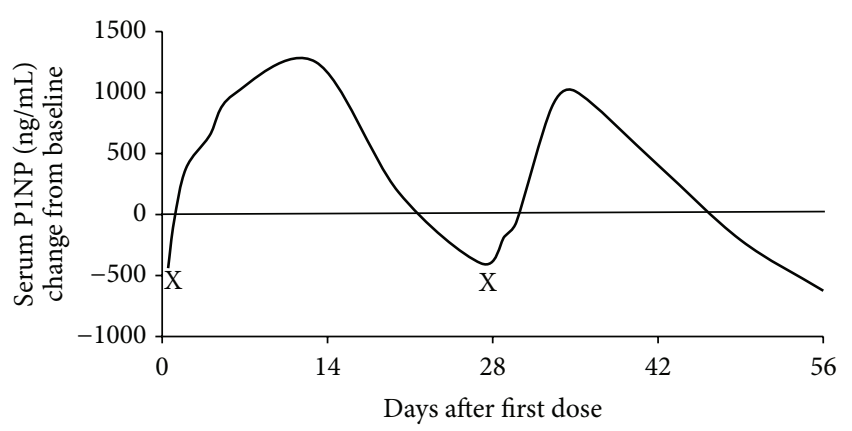

(a)

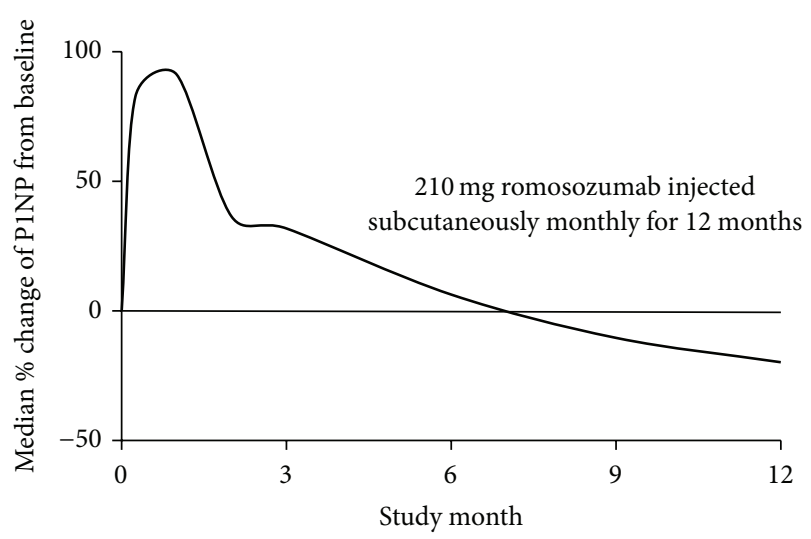

(b)

FIGURE 3: Change in serum concentrations of P1NP after multiple doses of anti-sclerostin antibodies in cynomolgus monkeys (a) and humans (b). (a) After $30 \mathrm{mg} / \mathrm{kg}$, subcutaneous dose of romosozumab P1NP levels (a marker of bone formation) increases in cynomolgus monkeys. Subsequently, P1NP levels decline before increasing subsequent to the second dose of romosozumab on day 29 (day of administration delineated by "X"). (b) This sharply contrasts with median levels of P1NP observed in human subjects after monthly doses of $210 \mathrm{mg}$ (total monthly dose) of blosozumab over a 12 -month period. Here a sharp increase in P1NP is observed after initial administration which peaks after the second subcutaneous injection of romosozumab at 1 month. Despite subsequent monthly doses, P1NP levels continue to decline and were observed to fall below baseline levels after 7 months, where levels remained until 12 months (demarcated the end of the study) [images adapted from [52] (a) and [55] (b)].

in human and nonhuman primate studies remain unclear. Similarly, the causes relating to the transient changes in biochemical markers of bone formation during blosozumab treatment have yet to be elucidated.

Some clarification may be sought in a recent study which assessed Scl-mAb $(25 \mathrm{mg} / \mathrm{kg})$ treatment administered once weekly for 6 or 26 weeks in ovx-rats [125]. It should be noted that this study did not involve the blosozumab antibody. Nevertheless, the study remains relevant given its investigation of the mechanistic effect on longer-term responses of osteoclasts, osteoblasts, and osteocytes subsequent to Scl-mAb treatment. Specifically, the authors observed that changes observed in bone resorption and formation at 6 weeks in ovx-Scl-mAb treated rats largely did not persist after
26 weeks. For instance, at 6-week Scl-mAb treatment induced a net reduction in bone resorption as evident from decreases in serum TRACP-5b and a reduction in the ex vivo capacity of marrow cells to differentiate into osteoclasts. Moreover, an $80 \%$ reduction in surface erosion within the vertebrae and trabecular and tibial endocortical surfaces compared to ovxvehicle samples was noted. At 26 weeks, however, the serum TRACP-5b and reduced ex vivo osteoclastic differentiation capacity of marrow cells were no longer evident. The observation that eroded surfaces remained over $80 \%$ lower in control groups nonetheless persisted.

Interestingly, at this time, the authors did not observe changes in RANKL or osteoprotegerin expression. As expected, Scl-mAb treatment induced significant increases in bone formation reflected by augmentations in serum P1NP and osteocalcin. Additionally, increases in the bone formation rate within trabecular, endocortical, and periosteal regions were observed. By week 26, however, only changes within the endocortical regions persevered while increases in skeletal mRNA of osteocytic genes were evident. In particular, SOST appeared to be markedly induced within the tibia and vertebral bone tissue [125]. One can cautiously speculate that the net increases in bone volume caused by the constitutive activation of Wnt-canonical signalling via Scl-mAb treatment may be synergistically counterregulated via increased osteocytic mediated sclerostin expression. Although these findings cannot be directly inferred to human outcomes, they may provide some insight as to the interstudy differences noted above. The potential for counterregulatory signalling to reduce bone formation will require further study to address its impact on longer-term treatments. To date, however, all published studies have demonstrated efficacy despite these observations. Taken together, these observations may better inform Scl-mAb treatment schedules. For now, the combination of Scl-mAb in conjunction with antiresorptive agents has demonstrated the most efficacy at trial. Therefore, it is likely that this approach will be the regimen that receives regulatory approval.

Generally, the safety profile of Scl-mAb treatment is positive, especially when compared to competitor osteoporotic therapeutics in development. For instance, the cathepsin $\mathrm{K}$ inhibitor MK 0822 (Merck, Odanacatib ${ }^{\mathrm{TM}}$ ) appears to increase, albeit not to statistically significant levels, the occurrence of atrial fibrillation and stroke in treated versus placebo groups [126]. These safety concerns with MK 0822 were only brought to light with the release of data from a phase III trial. Similarly, the full extent of the safety profiles of romosozumab and blosozumab cannot be fully determined from the comparatively small phase II studies.

\section{Summary and Outlook}

Compared to current anabolic market competitors such as teriparatide which is limited to 2 years' lifetime use, the substantial improvements in bone formation and microarchitecture subsequent to $\mathrm{Scl}-\mathrm{mAb}$ treatment have been unrivalled. Consequently, the results of clinical studies demonstrating the therapeutic potential of romosozumab in phase III trials are eagerly awaited. While the clinical data relating to efficacy 
appears clear, there remain significant questions relating to optimal treatment interval. For instance, given its apparent superiority to increase BMD, should Scl-mAb be the first line of treatment for osteoporotic patients? However, it remains to be established if Scl-mAb can be used for a long term in a cyclical manner either alone or in a complementary manner with antiresorptive options. Many studies have already been performed following Scl-mAb therapy with an antiremodelling agent. It is likely, therefore, that this regimen will receive regulatory approval. This approach may help address questions attesting to the tolerability of Scl-mAb and whether the emergence of neutralising antibodies may become more of an issue with increased exposure.

The emergence and clinical success of Scl-mAb have reinvigorated the therapeutic market for osteoporosis. Despite the many questions that remain, preclinical studies and published clinical trial results would imply that Scl-mAb will emerge as a dominant first-line treatment in the management of osteoporosis. Moreover, Scl-mAb is set to become a valuable tool in the development of international strategies to address the rise of osteoporosis and osteoporotic fracture management on a global scale.

\section{Competing Interests}

The authors declare that there is no conflict of interests regarding the publication of this paper.

\section{Acknowledgments}

Project no. S-13-130 is supported by the AO Foundation.

\section{References}

[1] E. M. Lewiecki, "Sclerostin monoclonal antibody therapy with AMG 785: a potential treatment for osteoporosis," Expert Opinion on Biological Therapy, vol. 11, no. 1, pp. 117-127, 2011.

[2] B. L. Clarke, "Anti-sclerostin antibodies: utility in treatment of osteoporosis," Maturitas, vol. 78, no. 3, pp. 199-204, 2014.

[3] L. Stodieck, AMGEN Countermeasures for Bone and Muscle Loss in Space and on Earth, 2013, http://www.slideshare.net/astrosociety/issrdc-2013-07170800stodieck.

[4] D. Padhi, G. Jang, B. Stouch, L. Fang, and E. Posvar, "Singledose, placebo-controlled, randomized study of AMG 785, a sclerostin monoclonal antibody," Journal of Bone and Mineral Research, vol. 26, no. 1, pp. 19-26, 2011.

[5] Osteoporosis and Osteopenia, 2013, http://decisionresources .com/Products-and-Services/Report?r=dbasmd 0213 .

[6] B. C. Silva and J. P. Bilezikian, "New approaches to the treatment of osteoporosis," Annual Review of Medicine, vol. 62, pp. 307322, 2011.

[7] T. D. Rachner, S. Khosla, and L. C. Hofbauer, "Osteoporosis: now and the future," The Lancet, vol. 377, no. 9773, pp. 12761287, 2011.

[8] K. M. P. Akesson, "Capture the fracture: a global campaign to the fragility fracture cycle," 2012, http://www..iofbonehealth.org/ capture-fracture-report-2012.

[9] O. Johnell, "The socioeconomic burden of fractures: today and in the 21st century," The American Journal of Medicine, vol. 103, no. 2, supplement 1, pp. S20-S26, 1997.
[10] G. Marongiu, M. Mastio, and A. Capone, "Current options to surgical treatment in osteoporotic fractures," Aging Clinical and Experimental Research, vol. 25, supplement 1, pp. S15-S17, 2013.

[11] S. C. Mears, "Management of severe osteoporosis in primary total hip arthroplasty," Current Translational Geriatrics and Experimental Gerontology Reports, vol. 2, no. 2, pp. 99-104, 2013.

[12] E. Hernlund, A. Svedbom, M. Ivergård et al., "Osteoporosis in the European Union: medical management, epidemiology and economic burden: a report prepared in collaboration with the International Osteoporosis Foundation (IOF) and the European Federation of Pharmaceutical Industry Associations (EFPIA)," Archives of Osteoporosis, vol. 8, no. 1-2, article 136, 2013.

[13] B. L. Riggs and L. J. Melton III, “The worldwide problem of osteoporosis: insights afforded by epidemiology," Bone, vol. 17, no. 5, supplement 1, pp. S505-S511, 1995.

[14] H. Hamersma, J. Gardner, and P. Beighton, "The natural history of sclerosteosis," Clinical Genetics, vol. 63, no. 3, pp. 192-197, 2003.

[15] A. G. Costa and J. P. Bilezikian, "Sclerostin: therapeutic horizons based upon its actions," Current Osteoporosis Reports, vol. 10, no. 1, pp. 64-72, 2012.

[16] W. Balemans, M. Ebeling, N. Patel et al., "Increased bone density in sclerosteosis is due to the deficiency of a novel secreted protein (SOST)," Human Molecular Genetics, vol. 10, no. 5, pp. 537-543, 2001.

[17] M. E. Brunkow, J. C. Gardner, J. Van Ness et al., "Bone dysplasia sclerosteosis results from loss of the SOST gene product, a novel cystine knot-containing protein," The American Journal of Human Genetics, vol. 68, no. 3, pp. 577-589, 2001.

[18] D. G. Winkler, M. K. Sutherland, J. C. Geoghegan et al., "Osteocyte control of bone formation via sclerostin, a novel BMP antagonist," The EMBO Journal, vol. 22, no. 23, pp. 62676276, 2003.

[19] C. Krause, O. Korchynskyi, K. de Rooij et al., "Distinct modes of inhibition by sclerostin on bone morphogenetic protein and Wnt signaling pathways," The Journal of Biological Chemistry, vol. 285, no. 53, pp. 41614-41626, 2010.

[20] A. R. Wijenayaka, M. Kogawa, H. P. Lim, L. F. Bonewald, D. M. Findlay, and G. J. Atkins, "Sclerostin stimulates osteocyte support of osteoclast activity by a RANKL-dependent pathway," PLoS ONE, vol. 6, no. 10, Article ID e25900, 2011.

[21] R. R. Recker, C. T. Benson, T. Matsumoto et al., "A randomized, double-blind phase 2 clinical trial of blosozumab, a sclerostin antibody, in postmenopausal women with low bone mineral density," Journal of Bone and Mineral Research, vol. 30, no. 2, pp. 216-224, 2015.

[22] X. Li, Y. Zhang, H. Kang et al., "Sclerostin binds to LRP5/6 and antagonizes canonical Wnt signaling," The Journal of Biological Chemistry, vol. 280, no. 20, pp. 19883-19887, 2005.

[23] M. V. Semenov and X. He, "LRP5 mutations linked to high bone mass diseases cause reduced LRP5 binding and inhibition by SOST," The Journal of Biological Chemistry, vol. 281, no. 50, pp. 38276-38284, 2006.

[24] R. Sugimura and L. Li, "Noncanonical Wnt signaling in vertebrate development, stem cells, and diseases," Birth Defects Research Part C: Embryo Today: Reviews, vol. 90, no. 4, pp. 243256, 2010.

[25] M. L. Johnson and M. A. Kamel, "The Wnt signaling pathway and bone metabolism," Current Opinion in Rheumatology, vol. 19, no. 4, pp. 376-382, 2007. 
[26] R. Baron and M. Kneissel, "WNT signaling in bone homeostasis and disease: from human mutations to treatments," Nature Medicine, vol. 19, no. 2, pp. 179-192, 2013.

[27] V. Krishnan, H. U. Bryant, and O. A. MacDougald, "Regulation of bone mass by Wnt signaling," The Journal of Clinical Investigation, vol. 116, no. 5, pp. 1202-1209, 2006.

[28] T. F. Day, X. Guo, L. Garrett-Beal, and Y. Yang, "Wnt/ $\beta$-catenin signaling in mesenchymal progenitors controls osteoblast and chondrocyte differentiation during vertebrate skeletogenesis," Developmental Cell, vol. 8, no. 5, pp. 739-750, 2005.

[29] D. A. Glass II, P. Bialek, J. D. Ahn et al., "Canonical Wnt signaling in differentiated osteoblasts controls osteoclast differentiation," Developmental Cell, vol. 8, no. 5, pp. 751-764, 2005.

[30] W. Qiu, L. Chen, and M. Kassem, "Activation of non-canonical Wnt/JNK pathway by Wnt3a is associated with differentiation fate determination of human bone marrow stromal (mesenchymal) stem cells," Biochemical and Biophysical Research Communications, vol. 413, no. 1, pp. 98-104, 2011.

[31] K. Maeda, N. Takahashi, and Y. Kobayashi, "Roles of Wnt signals in bone resorption during physiological and pathological states," Journal of Molecular Medicine, vol. 91, no. 1, pp. 15-23, 2013.

[32] H. Z. Ke, W. G. Richards, X. Li, and M. S. Ominsky, "Sclerostin and Dickkopf-1 as therapeutic targets in bone diseases," Endocrine Reviews, vol. 33, no. 5, pp. 747-783, 2012.

[33] Y. Gong, R. B. Slee, N. Fukai et al., "LDL receptor-related protein 5 (LRP5) affects bone accrual and eye development," Cell, vol. 107, no. 4, pp. 513-523, 2001.

[34] M. L. Johnson, G. Gong, W. Kimberling, S. M. Recker, D. B. Kimmel, and R. R. Recker, "Linkage of a gene causing high bone mass to human chromosome 11 (11q12-13)," American Journal of Human Genetics, vol. 60, no. 6, pp. 1326-1332, 1997.

[35] R. D. Little, C. Folz, S. P. Manning et al., "A mutation in the LDL receptor-related protein 5 gene results in the autosomal dominant high-bone-mass trait," The American Journal of Human Genetics, vol. 70, no. 1, pp. 11-19, 2002.

[36] M. Kato, M. S. Patel, R. Levasseur et al., "Cbfa1-independent decrease in osteoblast proliferation, osteopenia, and persistent embryonic eye vascularization in mice deficient in Lrp5, a Wnt coreceptor," Journal of Cell Biology, vol. 157, no. 2, pp. 303-314, 2002.

[37] P. Babij, W. Zhao, C. Small et al., "High bone mass in mice expressing a mutant LRP5 gene," Journal of Bone and Mineral Research, vol. 18, no. 6, pp. 960-974, 2003.

[38] W. Wei, D. Zeve, J. M. Suh et al., "Biphasic and dosage-dependent regulation of osteoclastogenesis by $\beta$-catenin," Molecular and Cellular Biology, vol. 31, no. 23, pp. 4706-4719, 2011.

[39] T. P. Hill, D. Später, M. M. Taketo, W. Birchmeier, and C. Hartmann, "Canonical Wnt $/ \beta$-catenin signaling prevents osteoblasts from differentiating into chondrocytes," Developmental Cell, vol. 8, no. 5, pp. 727-738, 2005.

[40] S. L. Holmen, C. R. Zylstra, A. Mukherjee et al., "Essential role of $\beta$-catenin in postnatal bone acquisition," The Journal of Biological Chemistry, vol. 280, no. 22, pp. 21162-21168, 2005.

[41] I. Kramer, C. Halleux, H. Keller et al., "Osteocyte Wnt/ $\beta$-catenin signaling is required for normal bone homeostasis," Molecular and Cellular Biology, vol. 30, no. 12, pp. 3071-3085, 2010.

[42] J. Albers, J. Keller, A. Baranowsky et al., "Canonical Wnt signaling inhibits osteoclastogenesis independent of osteoprotegerin," The Journal of Cell Biology, vol. 200, no. 4, pp. 537-549, 2013.
[43] T. A. Yorgan, S. Peters, A. Jeschke et al., "The anti-osteoanabolic function of sclerostin is blunted in mice carrying a high bone mass mutation of Lrp5," Journal of Bone and Mineral Research, vol. 30, no. 7, pp. 1175-1183, 2015.

[44] X. Li, M. S. Ominsky, Q.-T. Niu et al., "Targeted deletion of the sclerostin gene in mice results in increased bone formation and bone strength," Journal of Bone and Mineral Research, vol. 23, no. 6, pp. 860-869, 2008.

[45] G. J. Atkins, P. S. Rowe, H. P. Lim et al., "Sclerostin is a locally acting regulator of late-osteoblast/preosteocyte differentiation and regulates mineralization through a MEPE-ASARMdependent mechanism," Journal of Bone and Mineral Research, vol. 26, no. 7, pp. 1425-1436, 2011.

[46] P. Nioi, S. Taylor, R. Hu et al., "Transcriptional profiling of laser capture microdissected subpopulations of the osteoblast lineage provides insight into the early response to sclerostin antibody in rats," Journal of Bone and Mineral Research, vol. 30, no. 8, pp. 1457-1467, 2015.

[47] M. K. Robinson, J. Caminis, and M. E. Brunkow, "Sclerostin: how human mutations have helped reveal a new target for the treatment of osteoporosis," Drug Discovery Today, vol. 18, no. 13-14, pp. 637-643, 2013.

[48] M. J. C. Moester, S. E. Papapoulos, C. W. G. M. Löwik, and R. L. van Bezooijen, "Sclerostin: current knowledge and future perspectives," Calcified Tissue International, vol. 87, no. 2, pp. 99-107, 2010.

[49] E. M. Lewiecki, "Role of sclerostin in bone and cartilage and its potential as a therapeutic target in bone diseases," Therapeutic Advances in Musculoskeletal Disease, vol. 6, no. 2, pp. 48-57, 2014.

[50] X. Li, M. S. Ominsky, K. S. Warmington et al., "Sclerostin antibody treatment increases bone formation, bone mass, and bone strength in a rat model of postmenopausal osteoporosis," Journal of Bone and Mineral Research, vol. 24, no. 4, pp. 578-588, 2009.

[51] R. D. Ross, L. H. Edwards, A. S. Acerbo et al., "Bone matrix quality after sclerostin antibody treatment," Journal of Bone and Mineral Research, vol. 29, no. 7, pp. 1597-1607, 2014.

[52] M. S. Ominsky, F. Vlasseros, J. Jolette et al., "Two doses of sclerostin antibody in cynomolgus monkeys increases bone formation, bone mineral density, and bone strength," Journal of Bone and Mineral Research, vol. 25, no. 5, pp. 948-959, 2010.

[53] D. Padhi, M. Allison, A. J. Kivitz et al., "Multiple doses of sclerostin antibody romosozumab in healthy men and postmenopausal women with low bone mass: a randomized, doubleblind, placebo-controlled study," Journal of Clinical Pharmacology, vol. 54, no. 2, pp. 168-178, 2014.

[54] M. R. McClung, J. San Martin, P. D. Miller et al., "Opposite bone remodeling effects of teriparatide and alendronate in increasing bone mass," Archives of Internal Medicine, vol. 165, no. 15, pp. $1762-1768,2005$.

[55] M. R. McClung, A. Grauer, S. Boonen et al., "Romosozumab in postmenopausal women with low bone mineral density," The New England Journal of Medicine, vol. 370, no. 5, pp. 412-420, 2014.

[56] M. R. McClung, A. Chines, J. P. Brown et al., "OP0251 effects of 2 years of treatment with romosozumab followed by 1 year of denosumab or placebo in postmenopausal women with low bone mineral density," Annals of the Rheumatic Diseases, vol. 74, supplement 2, pp. 166-167, 2015.

[57] J. McColm, L. Hu, T. Womack, C. C. Tang, and A. Y. Chiang, "Single- and multiple-dose randomized studies of blosozumab, 
a monoclonal antibody against sclerostin, in healthy postmenopausal women," Journal of Bone and Mineral Research, vol. 29, no. 4, pp. 935-943, 2014.

[58] C. P. Recknor, R. R. Recker, C. T. Benson et al., "The effect of discontinuing treatment with blosozumab: follow-up results of a phase 2 randomized clinical trial in postmenopausal women with low bone mineral density," Journal of Bone and Mineral Research, vol. 30, no. 9, pp. 1717-1725, 2015.

[59] Novartis, Novartis Clinical Innovations Pipeline Annual Report, Pharmaceuticals, 2014.

[60] A. Morse, M. M. McDonald, N. H. Kelly et al., "Mechanical load increases in bone formation via a sclerostin-independent pathway," Journal of Bone and Mineral Research, vol. 29, no. 1, pp. 2456-2467, 2014.

[61] M.-K. Chang, I. Kramer, H. Keller et al., "Reversing LRP5dependent osteoporosis and SOST deficiency-induced sclerosing bone disorders by altering WNT signaling activity," Journal of Bone and Mineral Research, vol. 29, no. 1, pp. 29-42, 2014.

[62] A. Morse, N. Y. C. Yu, L. Peacock et al., "Endochondral fracture healing with external fixation in the Sost knockout mouse results in earlier fibrocartilage callus removal and increased bone volume fraction and strength," Bone, vol. 71, pp. 155-163, 2015.

[63] X. Li, M. S. Ominsky, K. S. Warmington et al., "Increased bone formation and bone mass induced by sclerostin antibody is not affected by pretreatment or cotreatment with alendronate in osteopenic, ovariectomized rats," Endocrinology, vol. 152, no. 9, pp. 3312-3322, 2011.

[64] J. S. Finkelstein, A. Hayes, J. L. Hunzelman, J. J. Wyland, H. Lee, and R. M. Neer, "The effects of parathyroid hormone, alendronate, or both in men with osteoporosis," The New England Journal of Medicine, vol. 349, no. 13, pp. 1216-1226, 2003.

[65] J. S. Finkelstein, B. Z. Leder, S.-A. M. Burnett et al., "Effects of teriparatide, alendronate, or both on bone turnover in osteoporotic men," The Journal of Clinical Endocrinology \& Metabolism, vol. 91, no. 8, pp. 2882-2887, 2006.

[66] L. Cui, H. Cheng, C. Song et al., "Time-dependent effects of sclerostin antibody on a mouse fracture healing model," Journal of Musculoskeletal \& Neuronal Interactions, vol. 13, no. 2, pp. 178-184, 2013.

[67] P. K. Suen, Y.-X. He, D. H. K. Chow et al., "Sclerostin monoclonal antibody enhanced bone fracture healing in an open osteotomy model in rats," Journal of Orthopaedic Research, vol. 32, no. 8, pp. 997-1005, 2014.

[68] M. S. Ominsky, C. Li, X. Li et al., "Inhibition of sclerostin by monoclonal antibody enhances bone healing and improves bone density and strength of nonfractured bones," Journal of Bone and Mineral Research, vol. 26, no. 5, pp. 1012-1021, 2011.

[69] M. M. McDonald, A. Morse, K. Mikulec et al., "Inhibition of sclerostin by systemic treatment with sclerostin antibody enhances healing of proximal tibial defects in ovariectomized rats," Journal of Orthopaedic Research, vol. 30, no. 10, pp. 15411548, 2012.

[70] M. U. Jawad, K. E. Fritton, T. Ma et al., "Effects of sclerostin antibody on healing of a non-critical size femoral bone defect," Journal of Orthopaedic Research, vol. 31, no. 1, pp. 155-163, 2013.

[71] C. S. Yee, L. Xie, S. Hatsell et al., "Sclerostin antibody treatment improves fracture outcomes in a type i diabetic mouse model," Bone, vol. 82, pp. 122-134, 2016.

[72] M. S. Virk, F. Alaee, H. Tang, M. S. Ominsky, H. Z. Ke, and J. R. Lieberman, "Systemic administration of sclerostin antibody enhances bone repair in a critical-sized femoral defect in a rat model," The Journal of Bone \& Joint Surgery-American Volume, vol. 95, no. 8, pp. 694-701, 2013.

[73] F. Alaee, M. S. Virk, H. Tang et al., "Evaluation of the effects of systemic treatment with a sclerostin neutralizing antibody on bone repair in a rat femoral defect model," Journal of Orthopaedic Research, vol. 32, no. 2, pp. 197-203, 2014.

[74] J. Levin, Acceleration of Fracture Healing with CDP7851/AMG785 Will Not Move into Phase 3, FierceBiotech, 2013, http://www .fiercebiotech.com/press-releases/acceleration-fracture-healingcdp7851amg785-will-not-move-phase-3.

[75] K. Sarahrudi, A. Thomas, C. Albrecht, and S. Aharinejad, "Strongly enhanced levels of sclerostin during human fracture healing," Journal of Orthopaedic Research, vol. 30, no. 10, pp. 1549-1555, 2012.

[76] F. Agholme, X. Li, H. Isaksson, H. Z. Ke, and P. Aspenberg, "Sclerostin antibody treatment enhances metaphyseal bone healing in rats," Journal of Bone and Mineral Research, vol. 25, no. 11, pp. 2412-2418, 2010.

[77] A. S. Virdi, M. Liu, K. Sena et al., "Sclerostin antibody increases bone volume and enhances implant fixation in a rat model," The Journal of Bone \& Joint Surgery-American Volume, vol. 94, no. 18, pp. 1670-1680, 2012.

[78] S. Liu, A. S. Virdi, K. Sena, and D. R. Sumner, "Sclerostin antibody prevents particle-induced implant loosening by stimulating bone formation and inhibiting bone resorption in a rat model," Arthritis and Rheumatism, vol. 64, no. 12, pp. 40124020, 2012.

[79] A. S. Virdi, J. Irish, K. Sena et al., "Sclerostin antibody treatment improves implant fixation in a model of severe osteoporosis," The Journal of Bone \& Joint Surgery - AmericanVolume, vol. 97, no. 2, pp. 133-140, 2015.

[80] A. Eddleston, M. Marenzana, A. R. Moore et al., "A short treatment with an antibody to sclerostin can inhibit bone loss in an ongoing model of colitis," Journal of Bone and Mineral Research, vol. 24, no. 10, pp. 1662-1671, 2009.

[81] X.-X. Chen, W. Baum, D. Dwyer et al., "Sclerostin inhibition reverses systemic, periarticular and local bone loss in arthritis," Annals of the Rheumatic Diseases, vol. 72, no. 10, pp. 1732-1736, 2013.

[82] C. Hamann, M. Rauner, Y. Höhna et al., "Sclerostin antibody treatment improves bone mass, bone strength, and bone defect regeneration in rats with type 2 diabetes mellitus," Journal of Bone and Mineral Research, vol. 28, no. 3, pp. 627-638, 2013.

[83] B. P. Sinder, M. M. Eddy, M. S. Ominsky, M. S. Caird, J. C. Marini, and K. M. Kozloff, "Sclerostin antibody improves skeletal parameters in a Brtl/+ mouse model of osteogenesis imperfecta," Journal of Bone and Mineral Research, vol. 28, no. 1, pp. 73-80, 2013.

[84] A. G. Costa, J. P. Bilezikian, and E. M. Lewiecki, "The potential use of antisclerostin therapy in chronic kidney diseasemineral and bone disorder," Current Opinion in Nephrology and Hypertension, vol. 24, no. 4, pp. 324-329, 2015.

[85] Y. Ren, X. Han, S. P. Ho et al., "Removal of SOST or blocking its product sclerostin rescues defects in the periodontitis mouse model," FASEB Journal, vol. 29, no. 7, pp. 2702-2711, 2015.

[86] A. Roschger, P. Roschger, P. Keplingter et al., "Effect of sclerostin antibody treatment in a mouse model of severe osteogenesis imperfecta," Bone, vol. 66, pp. 182-188, 2014. 
[87] D. Gatti, F. Antoniazzi, R. Prizzi et al., "Intravenous neridronate in children with osteogenesis imperfecta: a randomized controlled study," Journal of Bone and Mineral Research, vol. 20, no. 5, pp. 758-763, 2005.

[88] R. Sakkers, D. Kok, R. Engelbert et al., "Skeletal effects and functional outcome with olpadronate in children with osteogenesis imperfecta: a 2-year randomised placebo-controlled study," The Lancet, vol. 363, no. 9419, pp. 1427-1431, 2004.

[89] M. P. Whyte, W. H. McAlister, D. V. Novack, K. L. Clements, P. L. Schoenecker, and D. Wenkert, "Bisphosphonate-induced osteopetrosis: novel bone modeling defects, metaphyseal osteopenia, and osteosclerosis fractures after drug exposure ceases," Journal of Bone and Mineral Research, vol. 23, no. 10, pp. 1698-1707, 2008.

[90] L. M. Ward, F. Rauch, M. P. Whyte et al., "Alendronate for the treatment of pediatric osteogenesis imperfecta: a randomized placebo-controlled study," Journal of Clinical Endocrinology and Metabolism, vol. 96, no. 2, pp. 355-364, 2011.

[91] F. Rauch, C. F. Munns, C. Land, M. Cheung, and F. H. Glorieux, "Risedronate in the treatment of mild pediatric osteogenesis imperfecta: a randomized placebo-controlled study," Journal of Bone and Mineral Research, vol. 24, no. 7, pp. 1282-1289, 2009.

[92] A. D. Letocha, H. L. Cintas, J. F. Troendle et al., "Controlled trial of pamidronate in children with types III and IV osteogenesis imperfecta confirms vertebral gains but not short-term functional improvement," Journal of Bone and Mineral Research, vol. 20, no. 6, pp. 977-986, 2005.

[93] J. L. Vahle, M. Sato, G. G. Long et al., "Skeletal changes in rats given daily subcutaneous injections of recombinant human parathyroid hormone (1-34) for 2 years and relevance to human safety," Toxicologic Pathology, vol. 30, no. 3, pp. 312-321, 2002.

[94] J. C. Marini, E. Hopkins, F. H. Glorieux et al., "Positive linear growth and bone responses to growth hormone treatment in children with types III and IV osteogenesis imperfecta: high predictive value of the carboxyterminal propeptide of type I procollagen," Journal of Bone and Mineral Research, vol. 18, no. 2, pp. 237-243, 2003.

[95] M. Roudier, X. Li, Q.-T. Niu et al., "Sclerostin is expressed in articular cartilage but loss or inhibition does not affect cartilage remodeling during aging or following mechanical injury," Arthritis and Rheumatism, vol. 65, no. 3, pp. 721-731, 2013.

[96] M. H. Napimoga, C. Nametala, F. L. Da Silva et al., "Involvement of the Wnt- $\beta$-catenin signalling antagonists, sclerostin and dickkopf-related protein 1, in chronic periodontitis," Journal of Clinical Periodontology, vol. 41, no. 6, pp. 550-557, 2014.

[97] U. Kuchler, U. Y. Schwarze, T. Dobsak et al., "Dental and periodontal phenotype in sclerostin knockout mice," International Journal of Oral Science, vol. 6, no. 2, pp. 70-76, 2014.

[98] P. Han, S. Ivanovski, R. Crawford, and Y. Xiao, "Activation of the canonical Wnt signaling pathway induces cementum regeneration," Journal of Bone and Mineral Research, vol. 30, no. 7, pp. 1160-1174, 2015.

[99] A. D. Taut, Q. Jin, J.-H. Chung et al., "Sclerostin antibody stimulates bone regeneration after experimental periodontitis," Journal of Bone and Mineral Research, vol. 28, no. 11, pp. 23472356, 2013.

[100] H. Chen, X. Xu, M. Liu et al., "Sclerostin antibody treatment causes greater alveolar crest height and bone mass in an ovariectomized rat model of localized periodontitis," Bone, vol. 76, pp. 141-148, 2015.
[101] K. G. Murphy and J. C. Gunsolley, "Guided tissue regeneration for the treatment of periodontal intrabony and furcation defects. A systematic review," Annals of Periodontology, vol. 8, no. 1, pp. 266-302, 2003.

[102] S. Ivanovski, C. Vaquette, S. Gronthos, D. W. Hutmacher, and P. M. Bartold, "Multiphasic scaffolds for periodontal tissue engineering," Journal of Dental Research, vol. 93, no. 12, pp. 12121221, 2014.

[103] S. Pelletier, L. Dubourg, M.-C. Carlier, A. Hadj-Aissa, and D. Fouque, "The relation between renal function and serum sclerostin in adult patients with CKD," Clinical Journal of the American Society of Nephrology, vol. 8, no. 5, pp. 819-823, 2013.

[104] M. Kanbay, D. Siriopol, M. Saglam et al., "Serum sclerostin and adverse outcomes in nondialyzed chronic kidney disease patients," Journal of Clinical Endocrinology and Metabolism, vol. 99, no. 10, pp. E1854-E1861, 2014.

[105] V. M. Brandenburg, R. Kramann, R. Koos et al., "Relationship between sclerostin and cardiovascular calcification in hemodialysis patients: a cross-sectional study," BMC Nephrology, vol. 14, article 219, 2013.

[106] R. Kramann, V. M. Brandenburg, L. J. Schurgers et al., "Novel insights into osteogenesis and matrix remodelling associated with calcific uraemic arteriolopathy," Nephrology Dialysis Transplantation, vol. 28, no. 4, pp. 856-868, 2013.

[107] L. J. Schurgers, E. C. M. Cranenburg, and C. Vermeer, "Matrix Gla-protein: the calcification inhibitor in need of vitamin K," Thrombosis and Haemostasis, vol. 100, no. 4, pp. 593-603, 2008.

[108] M. Hayashi, I. Takamatsu, Y. Kanno, T. Yoshida, T. Abe, and Y. Sato, "A case-control study of calciphylaxis in Japanese endstage renal disease patients," Nephrology Dialysis Transplantation, vol. 27, no. 4, pp. 1580-1584, 2012.

[109] R. L. van Bezooijen, S. E. Papapoulos, and C. W. Löwik, "Bone morphogenetic proteins and their antagonists: the sclerostin paradigm," Journal of Endocrinological Investigation, vol. 28, no. 8, supplement, pp. 15-17, 2005.

[110] L. Desjardins, S. Liabeuf, R. B. Oliveira et al., "Uremic toxicity and sclerostin in chronic kidney disease patients," Nephrologie \& therapeutique, vol. 10, no. 6, pp. 463-470, 2014.

[111] L. Viaene, G. J. Behets, K. Claes et al., "Sclerostin: another bonerelated protein related to all-cause mortality in haemodialysis?" Nephrology Dialysis Transplantation, vol. 28, no. 12, pp. 30243030, 2013.

[112] C. Drechsler, P. Evenepoel, M. G. Vervloet et al., "High levels of circulating sclerostin are associated with better cardiovascular survival in incident dialysis patients: results from the NECOSAD study," Nephrology Dialysis Transplantation, vol. 30, no. 2, pp. 288-293, 2015.

[113] S. M. Moe, N. X. Chen, C. L. Newman et al., "Anti-sclerostin antibody treatment in a rat model of progressive renal osteodystrophy," Journal of Bone and Mineral Research, vol. 30, no. 3, pp. 499-509, 2015.

[114] C. L. Newman, N. X. Chen, E. Smith et al., "Compromised vertebral structural and mechanical properties associated with progressive kidney disease and the effects of traditional pharmacological interventions," Bone, vol. 77, pp. 50-56, 2015.

[115] V. Boschert, E.-M. Muth, A. Knappik, C. Frisch, and T. D. Mueller, "Crystallization and preliminary X-ray crystallographic analysis of the sclerostin-neutralizing Fab AbD09097," Acta Crystallographica Section F: Structural Biology Communications, vol. 71, part 4, pp. 388-392, 2015.

[116] Q. Yao, J. Ni, Y. Hou, L. Ding, L. Zhang, and H. Jiang, "Expression of sclerostin $\mathrm{scFv}$ and the effect of sclerostin scFv on healing 
of osteoporotic femur fracture in rats," Cell Biochemistry and Biophysics, vol. 69, no. 2, pp. 229-235, 2014.

[117] L. A. Johnson, Amgen, UCB Halt Testing of Drug for Fracture Healing, But Continue Testing for Osteoporosis, Associated Press, 2013, http://news.yahoo.com/amgen-ucb-halt-testing-fracture203100125.html.

[118] J. C. Gardner, R. L. van Bezooijen, B. Mervis et al., "Bone mineral density in sclerosteosis; affected individuals and gene carriers," The Journal of Clinical Endocrinology and Metabolism, vol. 90, no. 12, pp. 6392-6395, 2005.

[119] R. T. Moon, A. D. Kohn, G. V. De Ferrari, and A. Kaykas, "WNT and $\beta$-catenin signalling: diseases and therapies," Nature Reviews Genetics, vol. 5, no. 9, pp. 691-701, 2004.

[120] H. Clevers and R. Nusse, "Wnt/ $\beta$-catenin signaling and disease," Cell, vol. 149, no. 6, pp. 1192-1205, 2012.

[121] M. Kahn, "Can we safely target the WNT pathway?" Nature Reviews Drug Discovery, vol. 13, no. 7, pp. 513-532, 2014.

[122] M. Jia, S. Chen, B. Zhang et al., "Effects of constitutive $\beta$-catenin activation on vertebral bone growth and remodeling at different postnatal stages in mice," PLoS ONE, vol. 8, no. 9, Article ID e74093, 2013.

[123] S. Chen, J. Feng, Q. Bao et al., "Adverse effects of osteocytic constitutive activation of $B$-catenin on bone strength and bone growth," Journal of Bone and Mineral Research, vol. 30, no. 7, pp. 1184-1194, 2015.

[124] R. Baron and E. Hesse, "Update on bone anabolics in osteoporosis treatment: rationale, current status, and perspectives," The Journal of Clinical Endocrinology and Metabolism, vol. 97, no. 2, pp. 311-325, 2012.

[125] M. Stolina, D. Dwyer, Q.-T. Niu et al., “Temporal changes in systemic and local expression of bone turnover markers during six months of sclerostin antibody administration to ovariectomized rats," Bone, vol. 67, pp. 305-313, 2014.

[126] M. Co, Merck Announces Data from Pivotal Phase 3 Fracture Outcomes Study for Odanacatib, an Investigational Oral, Once-Weekly Treatment for Osteoporosis, 2014, http://www.mercknewsroom .com/news-release/research-and-development-news/merck-announces-data-pivotal-phase-3-fracture-outcomes-st. 


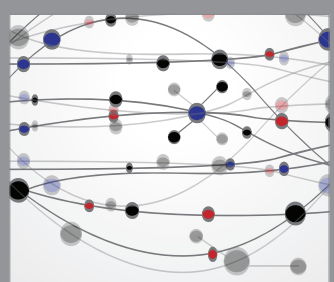

The Scientific World Journal
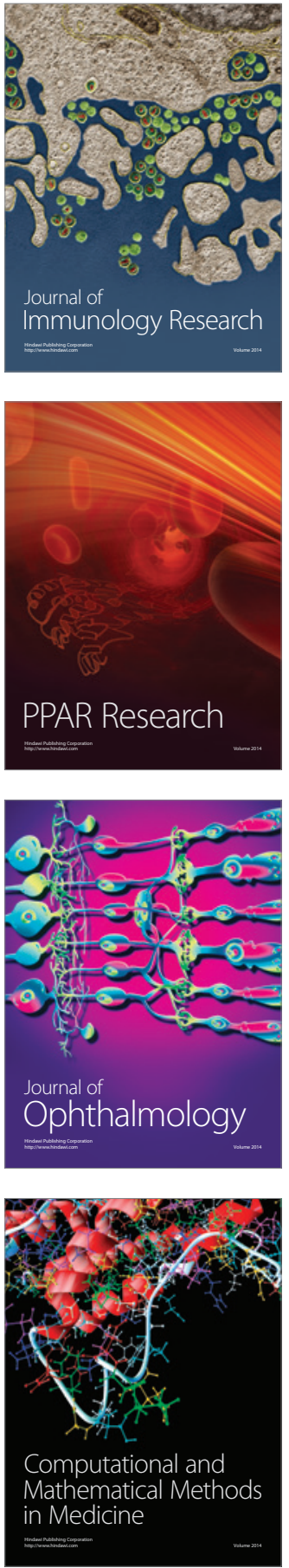

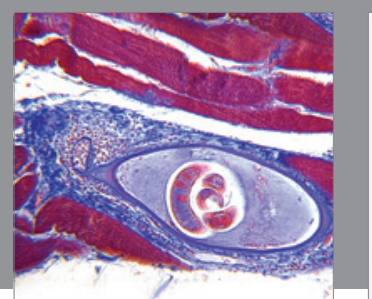

Gastroenterology Research and Practice

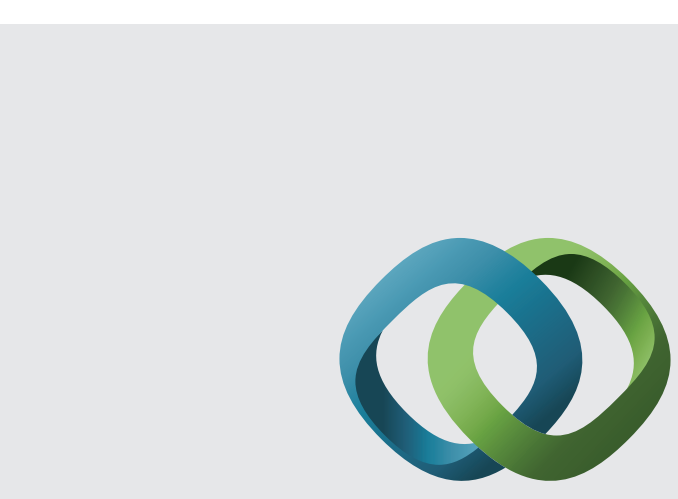

\section{Hindawi}

Submit your manuscripts at

http://www.hindawi.com
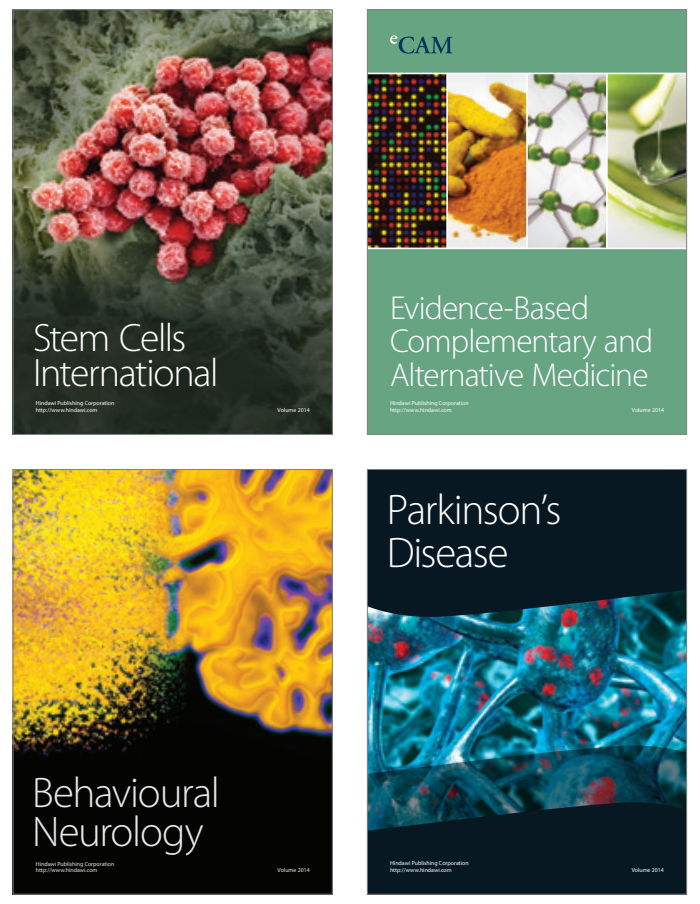
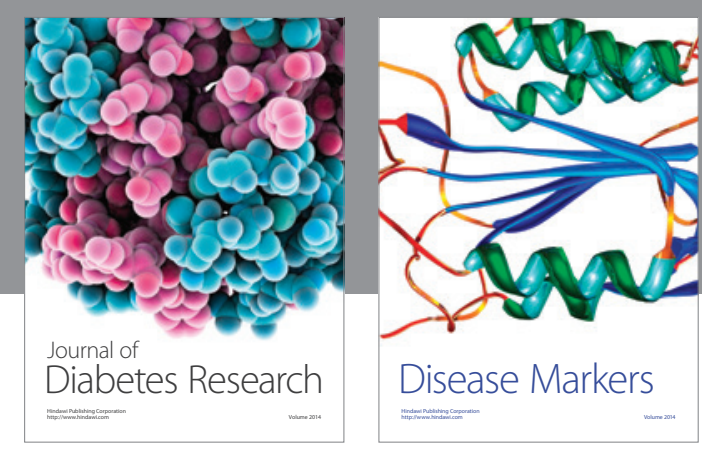

Disease Markers
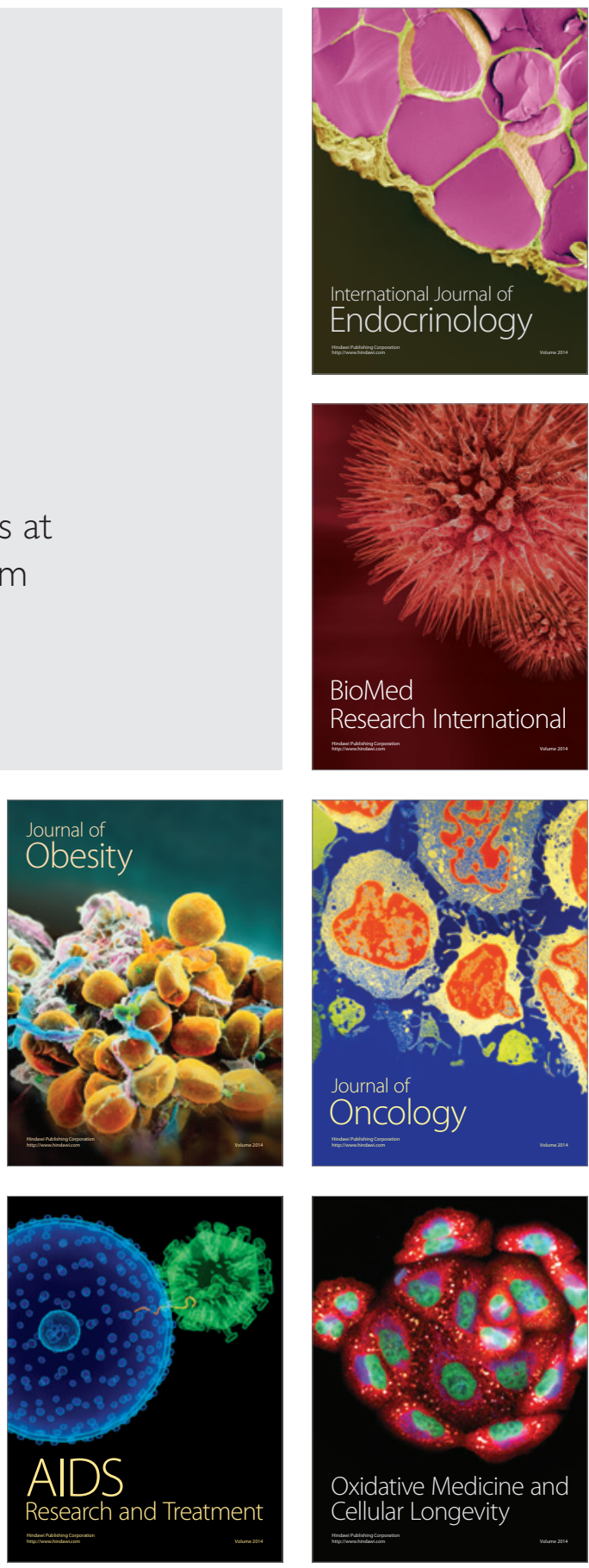